\title{
A Physical-layer Security Scheme Based on Cross-layer Cooperation in Dense Heterogeneous Networks
}

\author{
Zhang Bo ${ }^{1}$, Huang Kai-zhi ${ }^{1}$ and Chen Ya-jun ${ }^{1}$ \\ ${ }^{1}$ National Digital Switching System Engineering \& Technological R\&D Center, \\ Zheng Zhou 450001 - China \\ [e-mail: zb100403@126.com] [e-mail: huangkaizhi@tsinghua.org.cn] \\ *Corresponding author: Huang Kai-zhi
}

RReceived October 5, 2017; revised December 11, 2017; accepted January 2, 2018; published June 30, 2018

\begin{abstract}
In this paper, we investigate secure communication with the presence of multiple eavesdroppers (Eves) in a two-tier downlink dense heterogeneous network, wherein there is a macrocell base station (MBS) and multiple femtocell base stations (FBSs). Each base station (BS) has multiple users. And Eves attempt to wiretap a macrocell user (MU). To keep Eves ignorant of the confidential message, we propose a physical-layer security scheme based on cross-layer cooperation to exploit interference in the considered network. Under the constraints on the quality of service (QoS) of other legitimate users and transmit power, the secrecy rate of system can be maximized through jointly optimizing the beamforming vectors of MBS and cooperative FBSs. We explore the problem of maximizing secrecy rate in both non-colluding and colluding Eves scenarios, respectively. Firstly, in non-colluding Eves scenario, we approximate the original non-convex problem into a few semi-definite programs (SDPs) by employing the semi-definite relaxation (SDR) technique and conservative convex approximation under perfect channel state information (CSI) case. Furthermore, we extend the frame to imperfect CSI case and use the Lagrangian dual theory to cope with uncertain constraints on CSI. Secondly, in colluding Eves scenario, we transform the original problem into a two-tier optimization problem equivalently. Among them, the outer layer problem is a single variable optimization problem and can be solved by one-dimensional linear search. While the inner-layer optimization problem is transformed into a convex SDP problem with SDR technique and Charnes-Cooper transformation. In the perfect CSI case of both non-colluding and colluding Eves scenarios, we prove that the relaxation of SDR is tight and analyze the complexity of proposed algorithms. Finally, simulation results validate the effectiveness and robustness of proposed scheme.
\end{abstract}

Keywords: Dense heterogeneous netwoks, physical layer security, cooperative jamming, non-convex optimization, Semidefinite Relaxation (SDR)

A preliminary version of this paper appeared in IEEE VTC 2017, June 6-7, Sydeny. This version makes up for imperfect CSI case in non-colluding Eves scenario and both two CSI cases in colluding Eves scenario. This research was supported by some research grants 61701538, 61501516, and 61601514, which are from The Nationla Natural Science Fund, the Chinese government. 


\section{Introduction}

$\mathbf{W}_{\text {ith the huge growth of the smart devices, the next generation wireless systems have an }}$ increasing demand for extremely high data rate, ubiquitous coverage and reliable secrecy performance [1]. The dense deployment of network nodes, such as the FBSs underlaid in conventional macrocell, can provide better wireless coverage, higher efficiency of energy and spectrum [2]. Therefore, dense heterogeneous networks have attracted great research interest, and it is regarded as one of the most promising techniques in the next generation wireless systems $[3,4]$. However, compared with conventional single-tier cellular network, dense heterogeneous networks have a more open and diverse network architecture, which makes information exchange more susceptible to eavesdropping [5]. Recently, physical-layer security [6,7] has emerged as a complementary solution to conventional higher-layer cryptographic methods. Through exploiting the characteristics of wireless channels, such as fading, noise, and interference, it can guarantee the security of wireless communication, which is independent on complexity of computation $[8,9]$. Therefore, physical-layer security offers the opportunity to cope with the security problems appearing in dense heterogeneous networks.

Nevertheless, only a few literatures investigated the physical-layer security problems in dense heterogeneous networks. In [10], a physical-layer security model of dense heterogeneous networks was proposed and an expression of secrecy rate achieved was derived under this model. The authors in [11] proposed an access threshold-based secrecy mobile association policy, which associated each user with the BS providing the maximum truncated average received signal power beyond a threshold. And they derived a tractable expressions of secrecy probability and connection probability of a randomly located user respectively. In [12], a BS association constraint is imposed that the BSs providing an average received signal power(ARSP) at the legitimate user greater than a predetermined receiving threshold could be candidate serving BSs. And with this constraint, a closed-form expression for secrecy outage probability was derived. In [10], [11] and [12], only the performance analysis of physical-layer security in dense heterogeneous networks was considered, but how to improve the secrecy performance of system was not concerned.

The dense deployment of BSs makes the distance between them become small, and the hierarchical architecture is particularly suitable for a cooperation regime between two layers. Therefore, BS cooperation of cross-layer is regarded as a promising technique to improve the secrecy performance of system. The secrecy strategy for cooperative transmission and cooperative jamming in dense heterogeneous networks was studied in [13], and it analyzed the effect of artificial noise power ratio and the number of transmitting antennas on the secrecy performance of system. In [14], a dynamic coordinated multi-points(CoMP) scheme for the legitimate users was proposed to enhance secured coverage and the secured coverage probability of the scheme was analyzed. In this scheme, BSs providing an ARSP at the legitimate user greater than a predetermined receiving threshold were selected as serving BSs, and they would jointly transmit data for a legitimate user. According to the above analysis, it can be observed that the research of physical-layer security on cross-layer cooperation in dense heterogeneous networks is still largely missing. And the existing literatures studying the cooperative physical-layer security in traditional single-tier network may be of limited value because dense heterogeneous networks have a more complex architecture and more varies types of nodes. In dense heterogeneous networks, there is ubiquitous mutual interference of 
various type because of the densely overlaid network architecture and frequency multiplex. However, from the viewpoint of physical-layer security, the interference can be utilized through cross-layer cooperation between BSs, such as the MBS and FBSs, to deteriorate the received performance of eavesdroppers $[15,16]$. Therefore, it maybe feasible to design a physical-layer security scheme based on cross-layer cooperation.

In this paper, a two-tier downlink dense heterogeneous network is considered, wherein MBS and FBSs serve the corresponding legitimate MUs and femtocell users(FUs) respectively, and some MUs act maliciously as Eves to wiretap a legitimate MU. This means different legitimate users have corresponding secrecy levels, so some users may act as Eves for users having higher secrecy levels. So MBS can acquire the CSI of legitimate users and Eves. For this considered network, we explore a physical-layer security scheme based on cross-layer cooperation in both non-colluding Eves and colluding Eves scenarios respectively. FBSs adjacent to Eves are assigned to the same frequency resource as MBS, which means that they are cooperative FBSs. But the non-cooperative FBSs are assigned to resource orthogonal to that of MBS. Further, under the constraints on QoS of other MUs , FUs and transmit power, the secrecy rate of system can be maximized through jointly optimizing the beamforming vectors of MBS and cooperative FBSs. For clarity, the main contributions of this paper are summarized as follows:

1) In non-colluding Eves scenario, FBSs adjacent to the most dangerous Eve are cooperative FBSs. We first study the scheme under perfect CSI case. Utilizing SDR technique[17,18] and the successive convex approximation (SCA) algorithm [19], we recast the original problem of maximizing system secrecy rate, which is non-convex, into a convex SDP problem to obtain the optimal beamforming vectors. Further, we extend the frame to imperfect CSI case, where there are deterministic errors in channel covariance matrix. And we use the Lagrangian dual theory to deal with the uncertain constraints on CSI. In perfect CSI case, we can always prove that the relaxation of SDR is tight.

2) In colluding Eves scenario, FBSs adjacent to the middle dangerous Eve are cooperative FBSs. Through equivalent transformation and SDR technique, the original non-convex problem is transformed into a two-layer optimization problem to obtain the optimal beamforming vectors. Among them, the outer problem is a single variable optimization problem and can be solved by one-dimensional linear search. While the inner-layer optimization problem is transformed into a convex SDP problem using Charnes-Cooper [20] transformation. It should be pointed that the method of extending perfect CSI case to imperfect CSI case in non-colluding Eves scenario can also accommodate to colluding Eves scenario. Therefore, for concision of this paper, we only present the perfect CSI case of this scenario. Similarly, the rank of one beamforming vectors can always be acquired, which means the relaxation of SDR is tight.

3) We analyze and compare the complexity of algorithms to obtain the optimal beamforming vectors in two scenarios. In actual networks, we cannot know whether multiple Eves are colluding or not. To guarantee the security of system, it may be right to think they are colluding when applying the scheme. However, according to the analysis, we can know the complexity of algorithm in colluding Eves scenario is also higher. Therefore, the analysis of complexity may be suggestive in actual networks.

The remainder of this paper is organized as follows: Section 2 presents the system model. In Section 3, a physical-layer security scheme is proposed and how to obtain the optimal beamforming vectors of MBS and cooperative FBSs in two scenarios is investigated 
respectively. Then, the complexity of algorithms in two scenarios is analyzed. Simulation results are presented in Section 4. Finally, conclusion is drawn in Section 5.

\section{System Model}

As shown in Fig. 1, a two-tier downlink dense heterogeneous network is considered. There is an MBS at the center, and $M$ MUs are randomly distributed throughout the macrocell coverage area. $F$ FBSs are spatially distributed according to a homogeneous Poisson point process, and each FBS aims to serve $K$ FUs. $B$ potential Eves intend to wiretap the confidential message transmitted to a legitimate MU, and MBS knows the CSI of Eves [21]. MBS and each FBS are equipped with $N_{M}$ and $N_{F}$ antennas, respectively. Each legitimate user and each Eve is equipped with one antenna. MBS and each FBS service their corresponding users with beam-forming technology, so there is $N_{M}>M$ and $N_{F}>K$. To simplify the analysis, we assume that the transmit power of each FBS is fixed and equal, denoted by $P_{F}$. Similar to that, the transmit power of the MBS is assumed to be $P_{M}$. To interfere with Eves, the frequency resource occupied by the MBS was assigned to the FBSs which are adjacent to Eves. These FBSs are cooperative BSs in actual, while non-cooperative FBSs are assigned with orthogonal spectrum resources.

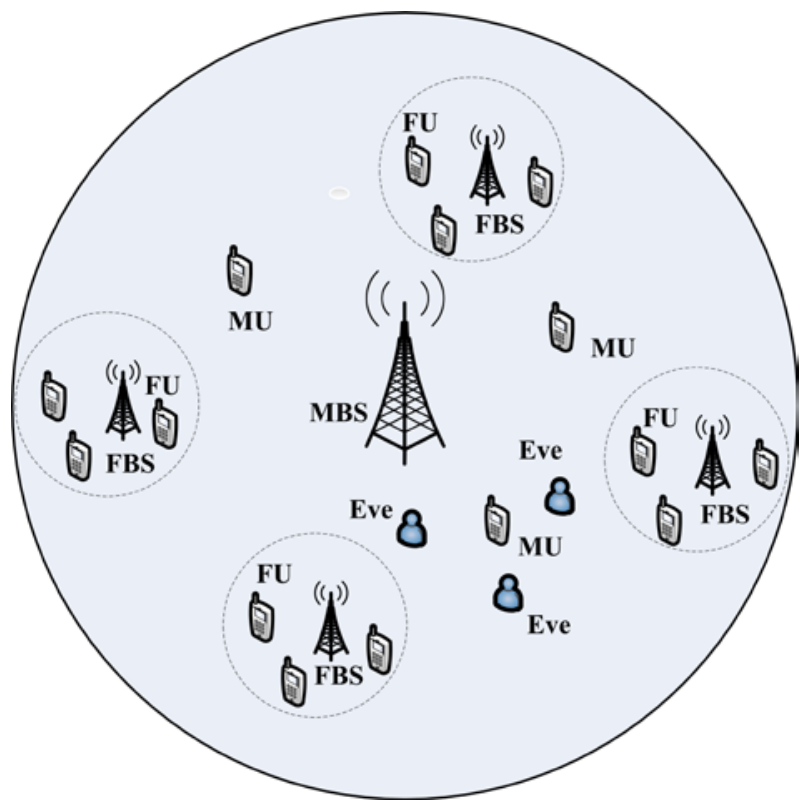

Fig. 1. A two-tier downlink dense heterogeneous network in the presence of multiple Eves

We assume that there are $N(1 \leq N \leq F)$ cooperative FBSs, and $\mathrm{MU}_{1}$ is wiretapped. Let us denote the $n$-th cooperative FBS as $\mathrm{FBS}_{n}$, the $m$-th $\mathrm{MU}$ as $\mathrm{MU}_{m}$, the $k$-th $\mathrm{FU}$ of $\mathrm{FBS}_{n}$ as $\mathrm{FU}_{n k}$ and the $b$-th Eve as $\mathrm{E}_{b}$ respectively. Then the received signal at $\mathrm{MU}_{m}, \mathrm{FU}_{n k}$ and $\mathrm{E}_{b}$ are respectively given by

$$
y_{m}=\boldsymbol{h}_{m} \boldsymbol{w}_{m} \mathrm{~s}_{m}+\sum_{p=1, p \neq m}^{M} \boldsymbol{h}_{m} \boldsymbol{w}_{p} \mathrm{~s}_{p}+\sum_{n=1}^{N} \sum_{k=1}^{K} \boldsymbol{h}_{n, m} \boldsymbol{w}_{n k} \mathrm{~s}_{n k}+\mathrm{n}_{m}, m \in[1, M]
$$




$$
\begin{gathered}
\boldsymbol{y}_{n k}=\boldsymbol{h}_{n, n k} \boldsymbol{w}_{n k} \boldsymbol{S}_{n k}+\sum_{t=1, t \neq k}^{K} \boldsymbol{h}_{n, n k} \boldsymbol{w}_{n t} \boldsymbol{S}_{n t}+\sum_{a=1, a \neq n}^{N} \sum_{t=1}^{K} \boldsymbol{h}_{a, n k} \boldsymbol{w}_{a t} \boldsymbol{S}_{a t}+\sum_{m=1}^{M} \boldsymbol{h}_{n k} \boldsymbol{w}_{m} \boldsymbol{s}_{m}+\mathrm{n}_{n k}, k \in[1, K] \\
\boldsymbol{y}_{E b}=\boldsymbol{h}_{E b} \boldsymbol{w}_{1} \mathrm{~S}_{1}+\sum_{m=2}^{M} \boldsymbol{h}_{E b} \boldsymbol{w}_{m} \boldsymbol{S}_{m}+\sum_{n=1}^{N} \sum_{k=1}^{K} \boldsymbol{h}_{n, E b} \boldsymbol{w}_{n k} S_{n k}+\mathrm{n}_{E b}, b \in[1, B]
\end{gathered}
$$

where $\boldsymbol{h}_{m} \in C^{1 \times N_{M}}$ and $\boldsymbol{h}_{n, m} \in C^{1 \times N_{F}}$ denote the channel vector from the MBS to $\mathrm{MU}_{m}$ and the channel vector from the $\mathrm{FBS}_{n}$ to $\mathrm{MU}_{m}$ respectively. $\boldsymbol{h}_{l, n k} \in C^{1 \times N_{F}}(l=n, a)$ denotes the channel vector from the $\mathrm{FBS}_{l}$ to $\mathrm{FU}_{n k}, \boldsymbol{h}_{n k} \in C^{1 \times N_{M}}$ denotes the channel vector from the MBS to $\mathrm{FU}_{n k} . \boldsymbol{h}_{E b} \in C^{1 \times N_{M}}$ and $\boldsymbol{h}_{n, E b} \in C^{1 \times N_{F}}$ denote the channel vector from the MBS to $\mathrm{E}_{b}$ and the channel vector from the $\mathrm{FBS}_{n}$ to $\mathrm{E}_{b} \cdot \boldsymbol{w}_{f} \in C^{N_{M} \times 1}(f=m, p)$ and $\boldsymbol{w}_{n k} \in C^{N_{F} \times 1}$ denote the beamforming vector from the MBS to $\mathrm{MU}_{f}$ and the beamforming vector from $\mathrm{FBS}_{n}$ to $\mathrm{FU}_{n k}$ respectively. $s_{i}(i=m, p)$ and $s_{n j}(j=k, t)$ denote the message symbol from the MBS to $\mathrm{MU}_{i}$ and the message symbol from the $\mathrm{FBS}_{n}$ to $\mathrm{FU}_{n j}$ respectively. $n_{m}, n_{n k}$ and $\mathrm{n}_{E b}$ denote the Gaussian noise at $\mathrm{MU}_{m}, \mathrm{FU}_{n k}$ and $\mathrm{E}_{b}$ respectively. To be specific, we assume all the message symbols and the Gaussian noise follow i.i.d $C N(0,1)$.

In non-colluding Eves scenario, the secrecy performance of system is determined by the most dangerous Eve. Then the secrecy rate of system is given by

$$
R_{S}(n c)=\left\{\log _{2}\left(1+\mathrm{SINR}_{1}\right)-\max _{b \in[1, B]}\left\{\log _{2}\left(1+\mathrm{SINR}_{E b}\right)\right\}\right\}^{+}
$$

where $\{a\}^{+}$denotes $\max \{a, 0\}, \operatorname{SINR}_{1}$ and $\operatorname{SINR}_{E b}$ denote the signal-to-interference-plus noise ratio (SINR) of $\mathrm{MU}_{1}$ and $\mathrm{E}_{b}$ respectively. They are respectively given by

$$
\begin{gathered}
\operatorname{SINR}_{1}=\frac{\left|\boldsymbol{h}_{1} \boldsymbol{w}_{1}\right|^{2}}{\sum_{m=2}^{M}\left|\boldsymbol{h}_{1} \boldsymbol{w}_{m}\right|^{2}+\sum_{n=1}^{N} \sum_{k=1}^{K}\left|\boldsymbol{h}_{n, 1} \boldsymbol{w}_{n k}\right|^{2}+1} \\
\operatorname{SINR}_{E b}=\frac{\left|\boldsymbol{h}_{E b} \boldsymbol{w}_{1}\right|^{2}}{\sum_{m=2}^{M}\left|\boldsymbol{h}_{E b} \boldsymbol{w}_{m}\right|^{2}+\sum_{n=1}^{N} \sum_{k=1}^{K}\left|\boldsymbol{h}_{n, E b} \boldsymbol{w}_{n k}\right|^{2}+1}
\end{gathered}
$$

In colluding Eves scenario, we can see multiple colluding single-antenna Eves as a multiple-antenna Eve $E_{B}$. Then the secrecy rate of system in this scenario can be given by

$$
R_{S}(c)=\left\{\log _{2}\left(1+\operatorname{SINR}_{1}\right)-\log _{2}\left(1+\operatorname{SINR}_{E B}\right)\right\}^{+}
$$

where $\operatorname{SINR}_{E B}$ denotes the SINR of $\mathrm{E}_{B}$, and it is given by

$$
\operatorname{SINR}_{E B}=\frac{\sum_{b=1}^{B}\left|\boldsymbol{h}_{E b} \boldsymbol{w}_{1}\right|^{2}}{\Gamma\left(\sum_{b=1}^{B} \sum_{m=2}^{M}\left|\boldsymbol{h}_{E b} \boldsymbol{w}_{m}\right|^{2}+\sum_{b=1}^{B} \sum_{n=1}^{N} \sum_{k=1}^{K}\left|\boldsymbol{h}_{n, E b} \boldsymbol{w}_{n k}\right|^{2}\right)+1}
$$

From (8), it is noted that multiple colluding Eves merged the energy of wiretapped signal, and they can eliminate a part of interference. $\Gamma^{\prime}$ is coefficient of eliminating interference, and $\Gamma=1-\Gamma^{\prime}\left(0 \leq \Gamma^{\prime} \leq 1\right)$. Additionally, from (4) (8), we can see that the secrecy rate of system and the received performance of other legitimate users( $\mathrm{MU}_{m}, m \neq 1$ and FUs) are all influenced 
by $\boldsymbol{w}_{m}$ and $\boldsymbol{w}_{n k}$ in both two scenarios. Therefore, the secrecy rate of system can be improved with cross-layer cooperation which optimizes the beamforming vectors of MBS and cooperative FBSs jointly [22].

\section{A Physical-layer Scheme Based on Cross-layer Cooperation}

In this section, we first investigate the proposed scheme in non-colluding Eves scenario. Then, we further study the scheme in colluding Eves scenario. Finally, We analyze complexity of the proposed algorithms to obtain the optimal beamforming vectors in two scenarios.

\subsection{Non-colluding Eves scenario}

In non-colluding Eves scenario, cooperative FBSs are the FBSs adjacent to the most dangerous Eve. Each cooperative FBS obtains its local CSI and then sends it to the MBS. As a result, global CSI becomes available at the MBS. Then, the MBS jointly optimizes the beamforming vectors with the aid of the global CSI to maximize the secrecy rate of system while guaranteeing the QoS of the other legitimate users. In the following, we first investigate the scheme in perfect CSI case, and then extend the frame to imperfect CSI case.

\subsubsection{Perfect CSI case}

According to the analysis in above sections, the original optimization problem is expressed as

$$
\begin{gathered}
\max _{\substack{\left\{\boldsymbol{w}_{m}\right\}_{m=1}^{M} \\
\left\{\left\{\boldsymbol{w}_{n k}\right\}_{k=1}^{K}\right\}_{1=1}^{N}}}\left\{R_{S}(n c)\right\} \\
\text { s.t. } \quad \sum_{m=1}^{M}\left\|\boldsymbol{w}_{m}\right\|^{2} \leq P_{M} \\
\sum_{k=1}^{K}\left\|\boldsymbol{w}_{n k}\right\|^{2} \leq P_{F}, n \in[1, N] \\
\operatorname{SINR}_{m} \geq \gamma_{m}, m \in[2, M] \\
\operatorname{SINR}_{n k} \geq \gamma_{n k}, n \in[1, N], k \in[1, K]
\end{gathered}
$$

where $\operatorname{SINR}_{m}$ and $\operatorname{SINR}_{n k}$ are the SINR of $\mathrm{MU}_{m}$ and $\mathrm{FU}_{n k}$ respectively. $\gamma_{m}$ and $\gamma_{n k}$ are QoS requirements of $\mathrm{MU}_{m}$ and $\mathrm{FU}_{n k}$ respectively. $\mathrm{SINR}_{m}$ and $\mathrm{SINR}_{n k}$ are respectively given by

$$
\begin{gathered}
\operatorname{SINR}_{m}=\frac{\left|\boldsymbol{h}_{m} \boldsymbol{w}_{m}\right|^{2}}{A_{m}}, m \in[1, M] \\
\operatorname{SINR}_{n k}=\frac{\left|\boldsymbol{h}_{n, n k} \boldsymbol{w}_{n k}\right|^{2}}{B_{n k}}
\end{gathered}
$$

where

$$
\begin{gathered}
A_{m}=\sum_{p=1, p \neq m}^{M}\left|\boldsymbol{h}_{m} \boldsymbol{w}_{p}\right|^{2}+\sum_{n=1}^{N} \sum_{k=1}^{K}\left|\boldsymbol{h}_{n, m} \boldsymbol{w}_{n k}\right|^{2}+1 \\
B_{n k}=\sum_{t=1, t \neq k}^{K}\left|\boldsymbol{h}_{n, n k} \boldsymbol{w}_{n t}\right|^{2}+\sum_{a=1, a \neq n}^{N} \sum_{t=1}^{K}\left|\boldsymbol{h}_{a, n k} \boldsymbol{w}_{a t}\right|^{2}+\sum_{m=1}^{M}\left|\boldsymbol{h}_{n k} \boldsymbol{w}_{m}\right|^{2}+1
\end{gathered}
$$

Problem (9) is non-convex and hence is very hard to solve. We introduce two slack variables $t_{1}$ and $t_{2}$, and then it can be equivalently transformed into 


$$
\begin{gathered}
\max _{\substack{\left\{w_{m}\right\}_{m=1}^{M} \\
\left\{\left\{w_{n k}\right\}_{k=1}^{K}\right\}_{n=1}^{N}}} \log _{2}\left(t_{1}\right)-\log _{2}\left(\frac{1}{t_{2}}\right) \\
\text { s.t. } \text { constraint (9b) constraint (9e) } \\
\operatorname{SINR}_{1} \geq t_{1}-1 \\
1+\operatorname{SINR}_{E b} \leq \frac{1}{t_{2}}
\end{gathered}
$$

It is observed that the equalities in (14f) and (14g) are tenable at the optimal solutions. Otherwise, the equalities are tenable through increasing $t_{1}$ and decreasing $1 / t_{2}$, and this operation will not change the optimal objective value. Therefore, problem (9) is equivalent to problem (14). To make problem (14) easier to tackle, we decompose (14f) and (14g) into several polynomial constraints by introducing auxiliary variables $t_{0}, t_{3}, t_{4}$. Then combined with (5) (6) , (10) (13) and SDR technique, problem (14) is reformulated as

$$
\begin{gathered}
\max _{\substack{\left\{\boldsymbol{w}_{m}\right\}_{m=1}^{M} \\
\left\{\left\{\boldsymbol{w}_{n k}\right\}_{k=1}^{K}\right\}_{n=1}^{N}}} t_{0} \\
\text { s.t. } \sum_{m=1}^{M} \operatorname{Tr}\left(\boldsymbol{W}_{m}\right) \leq P_{M} \\
\sum_{k=1}^{K} \operatorname{Tr}\left(\boldsymbol{W}_{n k}\right) \leq P_{F}, n \in[1, N] \\
\frac{\operatorname{Tr}\left(\boldsymbol{H}_{m} \boldsymbol{W}_{m}\right)}{A_{m}{ }^{\wedge}} \geq \gamma_{m}, m \in[2, M] \\
\frac{\operatorname{Tr}\left(\boldsymbol{H}_{n, n k} \boldsymbol{W}_{n k}\right)}{B_{n k}{ }^{\wedge} \geq \gamma_{n k}, n \in[1, N], k \in[1, K]} \\
\operatorname{Tr}^{M}\left(\boldsymbol{H}_{1} \boldsymbol{W}_{1}\right) \geq\left(t_{1}-1\right) t_{3} \\
\sum_{m=2}^{M} \operatorname{Tr}\left(\boldsymbol{H}_{1} \boldsymbol{W}_{m}\right)+\sum_{n=1}^{N} \sum_{k=1}^{K} \operatorname{Tr}\left(\boldsymbol{H}_{n, 1} \boldsymbol{W}_{n k}\right)+1 \leq t_{3} \\
\sum_{m=2}^{M} \operatorname{Tr}\left(\boldsymbol{H}_{E b} \boldsymbol{W}_{m}\right)+\sum_{n=1}^{N} \sum_{k=1}^{K} \operatorname{Tr}\left(\boldsymbol{H}_{n, E b} \boldsymbol{W}_{n k}\right)+1 \geq t_{2} t_{4} \\
\sum_{m=1}^{M} \operatorname{Tr}\left(\boldsymbol{H}_{E b} \boldsymbol{W}_{m}\right)+\sum_{n=1}^{N} \sum_{k=1}^{K} \operatorname{Tr}\left(\boldsymbol{H}_{n, E b} \boldsymbol{W}_{n k}\right)+1 \leq t_{4} \\
t_{0} \leq t_{1} t_{2}
\end{gathered}
$$

where $\boldsymbol{W}_{m}=\boldsymbol{w}_{m} \boldsymbol{w}_{m}{ }^{H}, \boldsymbol{W}_{n k}=\boldsymbol{w}_{n k} \boldsymbol{w}_{n k}{ }^{H}, \boldsymbol{H}_{m}=\boldsymbol{h}_{m}{ }^{H} \boldsymbol{h}_{m}, \boldsymbol{H}_{n, m}=\boldsymbol{h}_{n, m}{ }^{H} \boldsymbol{h}_{n, m}, \boldsymbol{H}_{n, n k}=\boldsymbol{h}_{n, n k}{ }^{H} \boldsymbol{h}_{n, n k}$, $\boldsymbol{H}_{n k}=\boldsymbol{h}_{n k}{ }^{H} \boldsymbol{h}_{n k}, \boldsymbol{H}_{E b}=\boldsymbol{h}_{E b}{ }^{H} \boldsymbol{h}_{E b}, \boldsymbol{H}_{n, E b}=\boldsymbol{h}_{n, E b}{ }^{H} \boldsymbol{h}_{n, E b} . A_{m}^{\wedge}$ and $B_{n k}{ }^{\wedge}$ are respectively expressed as

$$
\begin{gathered}
A_{m}^{\wedge}=\sum_{p=1, p \neq m}^{M} \operatorname{Tr}\left(\boldsymbol{H}_{m} \boldsymbol{W}_{p}\right)+\sum_{n=1}^{N} \sum_{k=1}^{K} \operatorname{Tr}\left(\boldsymbol{H}_{n, m} \boldsymbol{W}_{n k}\right)+1 \\
B_{n k}^{\wedge}=\sum_{t=1, t \neq k}^{K} \operatorname{Tr}\left(\boldsymbol{H}_{n, n k} \boldsymbol{W}_{n t}\right)+\sum_{a=1, a \neq n}^{N} \sum_{t=1}^{K} \operatorname{Tr}\left(\boldsymbol{H}_{a, n k} \boldsymbol{W}_{a t}\right)+\sum_{m=1}^{M} \operatorname{Tr}\left(\boldsymbol{H}_{n k} \boldsymbol{W}_{m}\right)+1
\end{gathered}
$$

Problem (15) is still non-convex because (15f) and (15h) have the form of $g(x, y)=x y$, which is quasi-concave. To solve this problem, we handle (15f) and (15h) with SCA algorithm. 
Specifically, we replace (15f) and (15h) with conservatively convex-constrained condition at first. Then, the problem (15) can be solved by solving a series of convex problem iteratively. For any $\chi>0$, we define the following function $[23,24]$

$$
f_{\chi}(x, y)=\frac{\chi}{2} x^{2}+\frac{1}{2 \chi} y^{2}
$$

It can be observed that $f_{\chi}(x, y)$ is always an upper estimate of $g(x, y)$. Further, $f_{\chi}(x, y)$ satisfies

$$
f_{\chi}(x, y)=g(x, y), \quad \nabla f_{\chi}(x, y)=\nabla g(x, y)
$$

when $\chi=y / x$. Obviously, if we replace $g(x, y)$ with $f_{x}(x, y)$, the constraints (15f) and (15h) can be conservatively approximated to several convex quadratic constraints. Therefore, in the $n$-th iterative approximation, (15f) and (15h) can be replaced by following constraints

$$
\begin{gathered}
\operatorname{Tr}\left(\boldsymbol{H}_{1} \boldsymbol{W}_{1}\right) \geq f_{\chi_{1}}{ }^{({ })}\left(t_{1}, t_{3}\right)-t_{3} \\
\sum_{m=2}^{M} \operatorname{Tr}\left(\boldsymbol{H}_{E b} \boldsymbol{W}_{m}\right)+\sum_{n=1}^{N} \sum_{k=1}^{K} \operatorname{Tr}\left(\boldsymbol{H}_{n, E b} \boldsymbol{W}_{n k}\right)+1 \geq f_{\chi_{2}}{ }^{\left({ }^{n}\right)}\left(t_{2}, t_{4}\right)
\end{gathered}
$$

where

$$
\begin{gathered}
f_{\chi_{1}}{ }^{(n)}\left(t_{1}, t_{3}\right)=\frac{\chi_{1}^{(n)}}{2} t_{1}^{2}+\frac{1}{2 \chi_{1}^{(n)}} t_{3}^{2} \\
f_{\chi_{2}}{ }^{(n)}\left(t_{2}, t_{4}\right)=\frac{\chi_{2}^{(n)}}{2} t_{2}{ }^{2}+\frac{1}{2 \chi_{2}^{(n)}} t_{4}^{2}
\end{gathered}
$$

where $\chi_{1}^{(n)}$ and $\chi_{2}^{(n)}$ are the ratio of $t_{3}$ to $t_{1}$ and the ratio of $t_{4}$ to $t_{2}$ in $(n-1)$-th iterative approximation respectively. For decreasing the computational complexity, we transform (15j) into a condition of Second Order Cone Programming (SOCP) $\left\|2 t_{0},\left(t_{1}-t_{2}\right)\right\| \leq t_{1}+t_{2}$ when $t_{1} \geq 0$ and $t_{2} \geq 0$ [25]. Then, in the $n$-th iterative approximation, problem (15) can be transformed into

$$
\begin{aligned}
& \max _{\substack{\left\{w_{m}\right\} \\
\left\{m=1 \\
\left\{\left\{w_{n k}\right\}_{k=1}^{N}\right\}_{n=1}^{N}\right.}} t_{0} \\
& \text { s.t. constraint(15b) (15e) } \\
& \operatorname{Tr}\left(\boldsymbol{H}_{1} \boldsymbol{W}_{1}\right) \geq \boldsymbol{f}_{\chi_{1}}\left(t_{1}, t_{3}\right)-t_{3} \\
& \sum_{m=2}^{M} \operatorname{Tr}\left(\boldsymbol{H}_{1} \boldsymbol{W}_{m}\right)+\sum_{n=1}^{N} \sum_{k=1}^{K} \operatorname{Tr}\left(\boldsymbol{H}_{n, 1} \boldsymbol{W}_{n k}\right)+1 \leq t_{3} \\
& \sum_{m=2}^{M} \operatorname{Tr}\left(\boldsymbol{H}_{E b} \boldsymbol{W}_{m}\right)+\sum_{n=1}^{N} \sum_{k=1}^{K} \operatorname{Tr}\left(\boldsymbol{H}_{n, E b} \boldsymbol{W}_{n k}\right)+1 \geq \boldsymbol{f}_{\chi_{2}}\left(t_{2}, t_{4}\right) \\
& \sum_{m=1}^{M} \operatorname{Tr}\left(\boldsymbol{H}_{E b} \boldsymbol{W}_{m}\right)+\sum_{n=1}^{N} \sum_{k=1}^{K} \operatorname{Tr}\left(\boldsymbol{H}_{n, E b} \boldsymbol{W}_{n k}\right)+1 \leq t_{4} \\
& \left\|\left[2 t_{0},\left(t_{1}-t_{2}\right)\right]\right\| \leq t_{1}+t_{2}
\end{aligned}
$$

Obviously, problem (24) is a tractable SDP problem that is convex and can be solved efficiently by using the existing solvers, such as CVX toolbox. It should be noted that the optimal solution to (24) may not be the optimal solution to (9) because the SDR technique is 
applied. However, we can show that the relaxation of $\boldsymbol{W}_{1}, \boldsymbol{W}_{m}$ and $\boldsymbol{W}_{n k}$ is always tight with the aid of the following proposition 1.

Proposition 1: If the problem (24) is feasible and that $\boldsymbol{W}_{1}^{\wedge}, \boldsymbol{W}_{m}^{\wedge}$ and $\boldsymbol{W}_{n k}^{\wedge}$ are the optimal solutions to (24). Then, they must be of rank one.

Proof: Please see Appendix.

According to Proposition 1, the optimal solutions $\boldsymbol{W}_{1}^{\wedge}, \boldsymbol{W}_{m}^{\wedge}$ and $\boldsymbol{W}_{n k}^{\wedge}$ of rank one to problem (9) can always be obtained through proposed Algorithm 1 in Table 1. The beamforming vectors $\boldsymbol{w}_{1}^{\wedge}, \boldsymbol{w}_{m}^{\wedge}$ and $\boldsymbol{w}_{n k}^{\wedge}$ can then be obtained directly from $\boldsymbol{W}_{1}^{\wedge}, \boldsymbol{W}_{m}^{\wedge}$ and $\boldsymbol{W}_{n k}^{\wedge}$ respectively using eigenvalue decomposition. At the same time, we can see that the solutions to $n$-th iteration are also those to $(n+1)$-th iteration. So the objective value of $(n+1)$-th iteration is no less than that of $n$-th iteration, which means that the optimal objective value $\left\{R_{s}^{(n)}\right\}$ is non-decreasing. Therefore, the secrecy rate of system must have an upper bound because the transmit power of system is limited, which means the Algorithm1 is converged.

Table 1. Algorithm 1

Algorithm1

The iterative algorithm for solving (9)

1) Initialization: Initializing $P_{M}, P_{F}, \gamma_{m}, \gamma_{n k}$, setting $n=0$, and selecting the values of $\left(\chi_{1}^{(n)}, \chi_{2}^{(n)}\right)$ belonging to the feasible set of $(24 \mathrm{a}) \sim(24 \mathrm{j})$;

2) Repeat:

Solving (24) and obtaining the optimal solutions of $\left(t_{1}, t_{2}, t_{3}, t_{4}\right)\left(t_{1}^{*}, t_{2}{ }^{*}, t_{3}{ }^{*}, t_{4}{ }^{*}\right)$;

$n=n+1$;

Updating $\chi_{1}^{(n)}=t_{1}^{*} / t_{3}^{*}, \chi_{2}^{(n)}=t_{2}^{*} / t_{4}^{*}$;

3) Loop termination: The algorithm converges or the required number of iterations;

4) Output: Local optimal solutions $\left(\boldsymbol{W}_{1}^{\wedge}, \boldsymbol{W}_{m}{ }^{\wedge}, \boldsymbol{W}_{n k}{ }^{\wedge}\right)$, optimal objective value $R_{s}^{\wedge}(n c)$.

\subsubsection{Imperfect CSI case}

In some circumstances, BS acquires the CSI of receivers in statistical feature of second order [26]. When there are errors in the estimation of channel covariance matrix, channel covariance matrix from transmitter to receiver can be modeled as $[27,28]$

$$
\boldsymbol{H}=\boldsymbol{h}^{H} \boldsymbol{h}=\hat{\boldsymbol{H}}+\Delta \boldsymbol{H}
$$

where $\boldsymbol{H}$ represents actual channel covariance matrix, $\hat{\boldsymbol{H}}$ represents the estimated channel covariance matrix, $\Delta \boldsymbol{H}$ represents the error associated with the channel. We consider the deterministic error model and $\Delta \boldsymbol{H}$ is in a spherical domain. According to semi-positive definiteness of channel covariance matrix, the uncertainty of the error matrix is defined as

$$
\Omega \triangleq\left\{\Delta \boldsymbol{H} \mid\|\Delta \boldsymbol{H}\|_{F} \leq \varepsilon, \hat{\boldsymbol{H}}+\Delta \boldsymbol{H} \geq \mathbf{0}, \Delta \boldsymbol{H}=\Delta \boldsymbol{H}^{H}\right\}
$$

where $\|\boldsymbol{X}\|_{F}$ represents the norm of matrix $\boldsymbol{X}, \varepsilon$ is the bound of error and $\varepsilon \geq 0$. Similar to (4), the secrecy rate of system in worst case can be expressed as 


$$
R_{S}(n c)=\min _{\substack{\Delta H_{m} \in \Omega_{m} \\ \Delta H_{n, m} \in \Omega_{n, m} \\ \Delta H_{n, k} \in \Omega_{n k} \\ \Delta \boldsymbol{H}_{a, n \in k} \in \Omega_{a, n k}}}\left\{\log _{2}\left(1+\operatorname{SINR}_{1}\right)-\log _{2}\left(1+\operatorname{SINR}_{E b}\right)\right\}^{+}, b \in[1, B], m \in[1, M], n \in[1, N], k \in[1, K]
$$

where $\Omega_{m}, \Omega_{n, m}, \Omega_{n k}$ and $\Omega_{a, n k}$ are the corresponding uncertainty of channel error matrix $\Delta \boldsymbol{H}_{m}, \Delta \boldsymbol{H}_{n, m}, \Delta \boldsymbol{H}_{n k}$ and $\Delta \boldsymbol{H}_{a, n k}$. Similar to problem (9), the problem of maximizing secrecy rate in this case can be transformed as

$$
\begin{aligned}
& \max _{\substack{\left\{w_{m}\right\}^{M} \\
\left\{\left\{=1 \\
\left\{\left\{w_{n k}\right\}_{k=1}^{N}\right\}_{n=1}^{N}\right.\right.}} t_{0} \\
& \text { s.t. constraint(15b) (15c) } \\
& \min _{\Delta \boldsymbol{H}_{m} \in \Omega_{m}} \operatorname{Tr}\left(\boldsymbol{H}_{m} \boldsymbol{W}_{m}\right) \geq \max _{\substack{\Delta \boldsymbol{H}_{m} \in \Omega_{m} \\
\Delta \boldsymbol{H}_{n, m} \in \Omega_{n, m}}}\left\{\gamma_{m} * A_{m}^{\wedge}\right\}, m \in[2, M] \\
& \min _{\substack{\Delta \boldsymbol{H}_{n, n k} \in \Omega_{n, n k} \\
\operatorname{Tr}}} \operatorname{Tr}\left(\boldsymbol{H}_{n, n k} \boldsymbol{W}_{n k}\right) \geq \max _{\substack{\Delta \boldsymbol{H}_{n, n k} \in \Omega_{n, n k} \\
\Delta \boldsymbol{H}_{n, k} \in \Omega_{a, n k} \\
\Delta \boldsymbol{H}_{n k} \in \Omega_{n k}}}\left(\gamma_{n k} * B_{n k}^{\wedge}\right), n \in[1, N], k \in[1, K] \\
& \min _{\Delta \boldsymbol{H}_{1} \in \Omega_{1}} \operatorname{Tr}\left(\boldsymbol{H}_{1} \boldsymbol{W}_{1}\right) \geq f_{\chi_{1}}\left(t_{1}, t_{3}\right)-t_{3} \\
& \max _{\substack{\Delta \boldsymbol{H}_{1} \in \Omega_{1} \\
\Delta \boldsymbol{H}_{n, 1} \in \Omega_{n, 1}}}\left\{\sum_{m=2}^{M} \operatorname{Tr}\left(\boldsymbol{H}_{1} \boldsymbol{W}_{m}\right)+\sum_{n=1}^{N} \sum_{k=1}^{K} \operatorname{Tr}\left(\boldsymbol{H}_{n, 1} \boldsymbol{W}_{n k}\right)+1\right\} \leq t_{3} \\
& \min _{\substack{\Delta \boldsymbol{H}_{E b} \in \Omega_{E b} \\
\Delta \boldsymbol{H}_{n, E b} \in \Omega_{n, E b}}}\left\{\sum_{m=2}^{M} \operatorname{Tr}\left(\boldsymbol{H}_{E b} \boldsymbol{W}_{m}\right)+\sum_{n=1}^{N} \sum_{k=1}^{K} \operatorname{Tr}\left(\boldsymbol{H}_{n, E b} \boldsymbol{W}_{n k}\right)+1\right\} \geq \boldsymbol{f}_{\chi_{2}}\left(t_{2}, t_{4}\right) \\
& \max _{\substack{\Delta \boldsymbol{H}_{E \in} \in \Omega_{E b} \\
\Delta \boldsymbol{H}_{n, E b} \in \Omega_{n, E b}}}\left\{\sum_{m=1}^{M} \operatorname{Tr}\left(\boldsymbol{H}_{E b} \boldsymbol{W}_{m}\right)+\sum_{n=1}^{N} \sum_{k=1}^{K} \operatorname{Tr}\left(\boldsymbol{H}_{n, E b} \boldsymbol{W}_{n k}\right)+1\right\} \leq t_{4} \\
& \left\|\left[2 t_{0},\left(t_{1}-t_{2}\right)\right]\right\| \leq t_{1}+t_{2}
\end{aligned}
$$

Problem (28) is an SDP problem that is convex. However, it should also be noted that there are infinite constraints in (28d) (28i) because of the error of channel covariance matrix. Now we apply Lemma 1 [28] and Lemma 2 [29] to deal with these infinite constraints.

Lemma 1: For any Hermitian matrix $\boldsymbol{R}, \boldsymbol{A}, \boldsymbol{\Delta} \in \mathrm{H}^{N}$, the optimization problem

$$
\begin{gathered}
\min \operatorname{Tr}(\boldsymbol{A}(\boldsymbol{R}+\boldsymbol{\Delta})) \\
\text { s.t. }\|\boldsymbol{\Delta}\| \leq \varepsilon, \boldsymbol{R}+\boldsymbol{\Delta} \geq \mathbf{0}
\end{gathered}
$$

is equivalent to the following optimization problem

$$
\begin{gathered}
\max _{\boldsymbol{S}}\left\{-\operatorname{Tr}(\boldsymbol{R}(\boldsymbol{S}-\boldsymbol{A}))-\chi\|\boldsymbol{S}-\boldsymbol{A}\|_{F}\right\} \\
\text { s.t. } \quad \boldsymbol{S} \geq \mathbf{0}
\end{gathered}
$$

Lemma 2: For any Hermitian matrix $\boldsymbol{R}, \boldsymbol{A}, \boldsymbol{\Delta} \in \mathrm{H}^{N}$, the optimal value of following optimization problem is $\operatorname{Tr}(\boldsymbol{R A})+\chi\|\boldsymbol{A}\|_{F}$.

$$
\begin{array}{ll} 
& \max \operatorname{Tr}(\boldsymbol{A}(\boldsymbol{R}+\boldsymbol{\Delta})) \\
\text { s.t. } & \|\boldsymbol{A}\| \leq \varepsilon, \boldsymbol{R}+\boldsymbol{\Delta} \geq \mathbf{0}, \boldsymbol{A} \geq \mathbf{0}
\end{array}
$$

According to the independence of channel and combining (16), we transform (28d) into 


$$
\min _{\Delta \boldsymbol{H}_{m} \in \Omega_{m}} \operatorname{Tr}\left(\boldsymbol{H}_{m} \boldsymbol{W}_{m}\right) \geq \gamma_{m} *\left\{\max _{\Delta \boldsymbol{H}_{m} \in \Omega_{m}} \sum_{p=1, p \neq m}^{M} \operatorname{Tr}\left(\boldsymbol{H}_{m} \boldsymbol{W}_{p}\right)+\max _{\Delta \boldsymbol{H}_{n, m} \in \Omega_{n, m}} \sum_{n=1}^{N} \sum_{k=1}^{K} \operatorname{Tr}\left(\boldsymbol{H}_{n, m} \boldsymbol{W}_{n k}\right)+1\right\}, m \in[2, \boldsymbol{M}]
$$

Adopting Lemma 1, we change (32) into

$$
\gamma_{m} *\left\{\max _{\Delta \boldsymbol{H}_{m} \in \Omega_{m}} \sum_{p=1, p \neq m}^{M} \operatorname{Tr}\left(\boldsymbol{H}_{m} \boldsymbol{W}_{p}\right)+\max _{\Delta \boldsymbol{H}_{n, m} \in \Omega_{n, m}} \sum_{n=1}^{N} \sum_{k=1}^{K} \operatorname{Tr}\left(\boldsymbol{H}_{n, m} \boldsymbol{W}_{n k}\right)+1\right\} \leq-\operatorname{Tr}\left(\hat{\boldsymbol{H}}_{m}\left(\boldsymbol{S}_{m}-\boldsymbol{W}_{m}\right)\right)-\chi_{m}\left\|\boldsymbol{S}_{m}-\boldsymbol{W}_{m}\right\|_{F}, m \in[2, \boldsymbol{M}]
$$

According to Lemma 2, we can see that

$$
\begin{aligned}
& \max _{\Delta \boldsymbol{H}_{m} \in \Omega_{m}} \sum_{p=1, p \neq m}^{M} \operatorname{Tr}\left(\boldsymbol{H}_{m} \boldsymbol{W}_{p}\right)=\sum_{p=1, p \neq m}^{M}\left\{\operatorname{Tr}\left(\hat{\boldsymbol{H}}_{m} \boldsymbol{W}_{p}\right)+\chi_{p}\left\|\boldsymbol{W}_{p}\right\|_{F}\right\} \\
& \max _{\Delta \boldsymbol{H}_{n, m} \in \Omega_{n, m}} \sum_{n=1}^{N} \sum_{k=1}^{K} \operatorname{Tr}\left(\boldsymbol{H}_{n, m} \boldsymbol{W}_{n k}\right)=\sum_{n=1}^{N} \sum_{k=1}^{K}\left\{\operatorname{Tr}\left(\hat{\boldsymbol{H}}_{n, m} \boldsymbol{W}_{n k}\right)+\chi_{n k}\left\|\boldsymbol{W}_{n k}\right\|_{F}\right\}
\end{aligned}
$$

So combining (33) and (34), we can transform (28d) into

$$
\begin{aligned}
& \gamma_{m} *\left\{\sum_{p=1, p \neq m}^{M}\{\right.\left.\left.\operatorname{Tr}\left(\hat{\boldsymbol{H}}_{m} \boldsymbol{W}_{p}\right)+\chi_{p}\left\|\boldsymbol{W}_{p}\right\|_{F}\right\}+\sum_{n=1}^{N} \sum_{k=1}^{K}\left\{\operatorname{Tr}\left(\hat{\boldsymbol{H}}_{n, m} \boldsymbol{W}_{n k}\right)+\chi_{n k}\left\|\boldsymbol{W}_{n k}\right\|_{F}\right\}+1\right\} \leq \\
&-\operatorname{Tr}\left(\hat{\boldsymbol{H}}_{m}\left(\boldsymbol{S}_{m}-\boldsymbol{W}_{m}\right)\right)-\chi_{m}\left\|\boldsymbol{S}_{m}-\boldsymbol{W}_{m}\right\|_{F}, m \in[2, M]
\end{aligned}
$$

At this time, it is observed that (35) is the deterministic form of (28d). Similarly, we can also transform (28e) (28i) into deterministic forms respectively. Then in the $n$-th iterative approximation, problem (28) can be transformed as

$$
\begin{aligned}
& \left.\max _{\substack{\left\{w_{m}\right\}_{m=1}^{M} \\
\left\{\left\{w_{n k}\right\}\right.}} t_{k=1}^{N}\right\}_{n=1}^{N} \\
& \text { s.t. constraint(15b) (15c) } \\
& \gamma_{m} *\left\{\sum_{p=1, p \neq m}^{M}\left\{\operatorname{Tr}\left(\hat{\boldsymbol{H}}_{m} \boldsymbol{W}_{p}\right)+\chi_{p}\left\|\boldsymbol{W}_{p}\right\|_{F}\right\}+\sum_{n=1}^{N} \sum_{k=1}^{K}\left\{\operatorname{Tr}\left(\hat{\boldsymbol{H}}_{n, m} \boldsymbol{W}_{n k}\right)+\chi_{n k}\left\|\boldsymbol{W}_{n k}\right\|_{F}\right\}+1\right\} \leq \\
& -\operatorname{Tr}\left(\hat{\boldsymbol{H}}_{m}\left(\boldsymbol{S}_{m}-\boldsymbol{W}_{m}\right)\right)-\chi_{m}\left\|\boldsymbol{S}_{m}-\boldsymbol{W}_{m}\right\|_{F}, m \in[2, \boldsymbol{M}] \\
& \gamma_{n k} *\left\{\sum_{t=1, t \neq k}^{K}\left\{\operatorname{Tr}\left(\hat{\boldsymbol{H}}_{n, n k} \boldsymbol{W}_{n t}\right)+\chi_{n t}\left\|\boldsymbol{W}_{n t}\right\|_{F}\right\}+\sum_{a=1, a \neq n}^{N} \sum_{t=1}^{K}\left\{\operatorname{Tr}\left(\hat{\boldsymbol{H}}_{a, n k} \boldsymbol{W}_{a t}\right)+\chi_{a t}\left\|\boldsymbol{W}_{a t}\right\|_{F}\right\}+\sum_{m=1}^{M}\left\{\operatorname{Tr}\left(\hat{\boldsymbol{H}}_{n k} \boldsymbol{W}_{m}\right)+\chi_{m}\left\|\boldsymbol{W}_{m}\right\|_{F}\right\}+1\right\} \leq \\
& -\operatorname{Tr}\left(\hat{\boldsymbol{H}}_{n, n k}\left(\boldsymbol{S}_{n k}-\boldsymbol{W}_{n k}\right)\right)-\chi_{n k}\left\|\boldsymbol{S}_{n k}-\boldsymbol{W}_{n k}\right\|_{F}, n \in[1, N], k \in[1, K] \\
& f_{\chi_{1}}\left(t_{1}, t_{3}\right)-t_{3} \leq-\operatorname{Tr}\left(\hat{\boldsymbol{H}}_{1}\left(\boldsymbol{S}_{1}-\boldsymbol{W}_{1}\right)\right)-\chi_{1}\left\|\boldsymbol{S}_{1}-\boldsymbol{W}_{1}\right\|_{F} \\
& \left\{\sum_{m=2}^{M}\left\{\operatorname{Tr}\left(\hat{\boldsymbol{H}}_{1} \boldsymbol{W}_{m}\right)+\chi_{m}\left\|\boldsymbol{W}_{m}\right\|_{F}\right\}+\sum_{n=1}^{N} \sum_{k=1}^{K}\left\{\operatorname{Tr}\left(\hat{\boldsymbol{H}}_{n, 1} \boldsymbol{W}_{n k}\right)+\chi_{n k}\left\|\boldsymbol{W}_{n k}\right\|_{F}\right\}+1\right\} \leq t_{3} \\
& \left\{\sum_{m=2}^{M}\left\{\operatorname{Tr}\left(\hat{\boldsymbol{H}}_{E b} \boldsymbol{W}_{m}\right)+\chi_{m}\left\|\boldsymbol{W}_{m}\right\|_{F}\right\}+\sum_{n=1}^{N} \sum_{k=1}^{K}\left\{\operatorname{Tr}\left(\hat{\boldsymbol{H}}_{n, E b} \boldsymbol{W}_{n k}\right)+\chi_{n k}\left\|\boldsymbol{W}_{n k}\right\|_{F}\right\}+1\right\} \geq f_{\chi_{2}}\left(t_{2}, t_{4}\right)
\end{aligned}
$$




$$
\begin{gathered}
\left\{\sum_{m=1}^{M}\left\{\operatorname{Tr}\left(\hat{\boldsymbol{H}}_{E b} \boldsymbol{W}_{m}\right)+\chi_{m}\left\|\boldsymbol{W}_{m}\right\|_{F}\right\}+\sum_{n=1}^{N} \sum_{k=1}^{K}\left\{\operatorname{Tr}\left(\hat{\boldsymbol{H}}_{n, E b} \boldsymbol{W}_{n k}\right)+\chi_{n k}\left\|\boldsymbol{W}_{n k}\right\|_{F}\right\}+1\right\} \leq t_{4} \\
\left\|\left[2 t_{0},\left(t_{1}-t_{2}\right)\right]\right\| \leq t_{1}+t_{2}
\end{gathered}
$$

Problem (36) is a convex SDP problem that can be efficiently solved. The algorithm for solving (28) is similar to algorithm 1, and it only needs to replace (24a) (24j) in Algorithm 1 with (36a) $(36 \mathrm{j})$. If $\operatorname{rank}\left(\boldsymbol{W}_{1}^{\wedge}\right), \operatorname{rank}\left(\boldsymbol{W}_{m}^{\wedge}\right)$ or $\operatorname{rank}\left(\boldsymbol{W}_{n k}^{\wedge}\right)=1$, we can get the beamforming vectors $\boldsymbol{w}_{1}^{\wedge}, \boldsymbol{w}_{m}^{\wedge}$ and $\boldsymbol{w}_{n k}^{\wedge}$ by eigenvalue decomposition. Otherwise, we can adopt some rank-one approximation procedures, such as Gaussian randomization [30] to obtain $\boldsymbol{w}_{1}^{\wedge}, \boldsymbol{w}_{m}^{\wedge}$ and $\boldsymbol{w}_{n k}^{\wedge}$, respectively.

\subsection{Colluding Eves scenario}

In colluding Eves scenario, cooperative FBSs are adjacent to the middle dangerous Eve. Then the original optimization problem is expressed as

$$
\begin{aligned}
& \max _{\substack{\left\{\boldsymbol{w}_{m}\right\}_{m=1}^{M} \\
\left\{\left\{w_{n k}\right\}_{k=1}^{N}\right\}_{n=1}^{N}}}\left\{\log _{2}\left(1+\operatorname{SINR}_{1}\right)-\log _{2}\left(1+\operatorname{SINR}_{E B}\right)\right\} \\
& \text { s.t. constraint }(9 \mathrm{~b}) \sim(9 \mathrm{e}) \quad \text { (37b ) (37e) }
\end{aligned}
$$

Problem (37) is non-convex. Introducing a slack variable $\operatorname{SINR}_{\text {Esum }}=\tau$ and combining SDR technique, we reformulate problem (37) as

$$
\begin{gathered}
\max _{\substack{\left\{\boldsymbol{w}_{m}\right\}_{m=1}^{M} \\
\left\{\left\{\boldsymbol{w}_{k k}\right\}_{k=1}^{N}\right\}_{n=1}^{N}}} \frac{1}{1+\tau}\left[1+\frac{\operatorname{Tr}\left(\boldsymbol{H}_{1} \boldsymbol{W}_{1}\right)}{A_{1}^{\wedge}}\right] \\
\text { s.t. constraint(15b) }(15 \mathrm{e}) \\
\frac{\sum_{b=1}^{B} \operatorname{Tr}\left(\boldsymbol{H}_{E b} \boldsymbol{W}_{1}\right)}{C_{E B}{ }^{\wedge}} \leq \tau
\end{gathered}
$$

where

$$
C_{E B}^{\wedge}=\Gamma\left(\sum_{b=1}^{B} \sum_{m=2}^{M} \operatorname{Tr}\left(\boldsymbol{H}_{E b} \boldsymbol{W}_{m}\right)+\sum_{b=1}^{B} \sum_{n=1}^{N} \sum_{k=1}^{K} \operatorname{Tr}\left(\boldsymbol{H}_{n, E b} \boldsymbol{W}_{n k}\right)\right)+1, b \in[1, B]
$$

Problem (38) is still non-convex. So we further introduce an auxiliary variable $G(\tau)=\operatorname{Tr}\left(\boldsymbol{H}_{1} \boldsymbol{W}_{1}\right) / A_{1}^{\wedge}$ and transformed it equivalently into a two-layer optimization problem. Among them, the outer optimization problem is a one-dimensional line search problem with $\tau$, i.e.,

$$
\begin{gathered}
\max _{\tau} \frac{1+G(\tau)}{1+\tau} \\
\text { s.t. } \tau_{\min } \leq \tau \leq \tau_{\max }
\end{gathered}
$$

Obviously, $\tau_{\min }=0$ and $\tau \leq \operatorname{SINR}_{1}$ because the secrecy rate of system is non-negative. Further, we konw $0 \leq \tau \leq \mathrm{P}_{\mathrm{M}} \operatorname{Tr}\left(\boldsymbol{H}_{1}\right)$. When $\tau$ is fixed, the internal optimization problem is

$$
\max _{\substack{\left\{\boldsymbol{w}_{m}\right\}_{m=1}^{M} \\\left\{\left\{\boldsymbol{w}_{n k}\right\}_{k=1}^{N}\right\}_{n=1}^{N}}} \frac{\operatorname{Tr}\left(\boldsymbol{H}_{\mathbf{1}} \boldsymbol{W}_{\mathbf{1}}\right)}{A_{1}^{\wedge}}
$$




$$
\text { s.t. constraint (39b) (39f) }
$$

The objective function in (41a) is a fractional function and thus it is quasi-convex. Then, we use Charnes-Cooper transformation to transform it into a linear one. To be specific, introducing auxiliary variables $\boldsymbol{X}_{1} \geq 0, \boldsymbol{X}_{m} \geq 0, \boldsymbol{X}_{n k} \geq 0$ and $\zeta>0$, we can express the beamforming vectors respectively as $\boldsymbol{W}_{1}=\boldsymbol{X}_{1} / \zeta, \boldsymbol{W}_{m}=\boldsymbol{X}_{m} / \zeta, \boldsymbol{W}_{n k}=\boldsymbol{X}_{n k} / \zeta$. Then problem (41) can be equivalently transformed into

$$
\begin{gathered}
\max _{\substack{\left\{\boldsymbol{x}_{m}\right\}_{m=1}^{M}, \zeta \\
\left\{\left\{\boldsymbol{x}_{n k}\right\}_{k=1}^{k}\right\}_{n=1}^{N}}} \operatorname{Tr}\left(\boldsymbol{H}_{1} \boldsymbol{X}_{1}\right) \\
\text { s.t. } \quad \sum_{m=1}^{M} \operatorname{Tr}\left(\boldsymbol{X}_{m}\right) \leq P_{M} \zeta \\
\sum_{k=1}^{K} \operatorname{Tr}\left(\boldsymbol{X}_{n k}\right) \leq P_{F} \zeta, n \in[1, N] \\
\operatorname{Tr}\left(\boldsymbol{H}_{m} \boldsymbol{X}_{m}\right) \geq \gamma_{m}\left(\sum_{p=1, p \neq m}^{M} \operatorname{Tr}\left(\boldsymbol{H}_{m} \boldsymbol{X}_{p}\right)+\sum_{n=1}^{N} \sum_{k=1}^{K} \operatorname{Tr}\left(\boldsymbol{H}_{n, m} \boldsymbol{X}_{n k}\right)+\zeta\right) \\
\operatorname{Tr}\left(\boldsymbol{H}_{n, n k} \boldsymbol{X}_{n k}\right) \geq \gamma_{n k}\left(\sum_{t=1, t \neq k}^{K} \operatorname{Tr}\left(\boldsymbol{H}_{n, n k} \boldsymbol{X}_{n t}\right)+\sum_{a=1, a \neq n}^{N} \sum_{t=1}^{K} \operatorname{Tr}\left(\boldsymbol{H}_{a, n k} \boldsymbol{X}_{a t}\right)+\sum_{m=1}^{M} \operatorname{Tr}\left(\boldsymbol{H}_{n k} \boldsymbol{X}_{m}\right)+\zeta\right) \\
\sum_{b=1}^{B} \operatorname{Tr}\left(\boldsymbol{H}_{E b} \boldsymbol{X}_{1}\right) \leq \tau\left(\Gamma\left(\sum_{b=1}^{B} \sum_{m=2}^{M} \operatorname{Tr}\left(\boldsymbol{H}_{E b} \boldsymbol{X}_{m}\right)+\sum_{b=1}^{B} \sum_{n=1}^{N} \sum_{k=1}^{K} \operatorname{Tr}\left(\boldsymbol{H}_{n, E b} \boldsymbol{X}_{n k}\right)\right)+\zeta\right), b \in[2, B] \\
\sum_{m=2}^{M} \operatorname{Tr}\left(\boldsymbol{H}_{m} \boldsymbol{X}_{m}\right)+\sum_{n=1}^{N} \sum_{k=1}^{K} \operatorname{Tr}\left(\boldsymbol{H}_{n, m} \boldsymbol{X}_{n k}\right)+\zeta=1 \\
\boldsymbol{X}_{1} \geq 0, \boldsymbol{X}_{m} \geq 0, \boldsymbol{X}_{n k} \geq 0, \zeta \geq 0
\end{gathered}
$$

Observing (42h), we can see that there are some transformations that may change the range of solutions in problem (42). But in fact, the optimal beamforming vectors must be greater than zero. Because if $\zeta=0$, the beamforming vectors must be zero. This means that the QoS requirements of MUs and FUs cannot be satisfied and is not identical with (41d), (41e). Therefore, transformations of (41h) have no influence on the optimal solutions. Up to now, the inner optimization problem has been transformed into an SDP problem that is convex and can be efficiently solved. It should be noted that the application of SDR technique may lead the optimal solutions to relaxed problem are not the optimal solutions to (38). However, we can show that the relaxations of $\boldsymbol{W}_{1}, \boldsymbol{W}_{m}$ and $\boldsymbol{W}_{n k}$ are always tight with the aid of the following proposition 2.

Proposition 2: If problem (42) is feasible and that $\boldsymbol{W}_{1}^{*}, \boldsymbol{W}_{m}^{*}$ and $\boldsymbol{W}_{n k}^{*}$ are the optimal solutions to problem (42). Then, they must be of rank one.

Proof: Please see Appendix.

Based on Proposition 2, the optimal solutions $\boldsymbol{W}_{1}^{*}, \boldsymbol{W}_{m}^{*}$ and $\boldsymbol{W}_{n k}^{*}$ of rank one for problem (37) can always be obtained. For any fixed $\tau \in\left[\tau_{\min }, \tau_{\max }\right]$, the optimal objective value of inner problem $G(\tau)$ can be acquired by solving (42). Then, the optimal objective value of outer problem can be acquired with one-dimension linear search method such as the Golden Section Search. The proposed algorithm of solving (37) is summarized in Table 2. The beamforming vectors $\boldsymbol{w}_{1}^{*}, \boldsymbol{w}_{m}^{*}$ and $\boldsymbol{w}_{n k}^{*}$ can then be obtained directly from $\boldsymbol{W}_{1}^{*}, \boldsymbol{W}_{m}^{*}$ and $\boldsymbol{W}_{n k}^{*}$ respectively using eigenvalue decomposition. 
Table 2. Algorithm 2

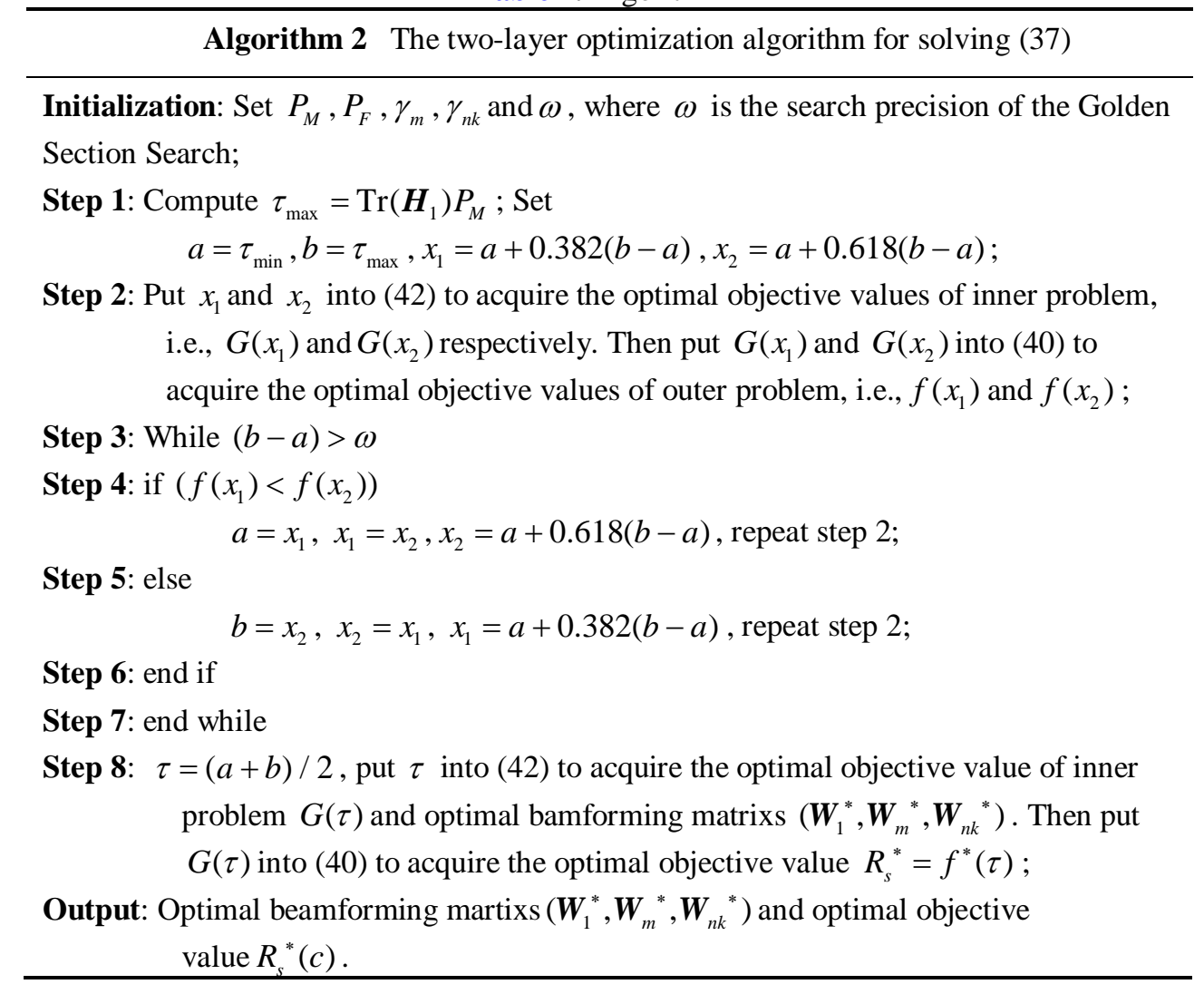

\subsection{Analysis of complexity}

According to the above analysis, the error of channel covariance matrix has the same influence on algorithm 1 and algorithm 2. So we only analyze and compare the complexity of these two algorithms in perfect CSI case, and the result of comparison also accommodates to imperfect CSI case. Observing algorithm 1 and algorithm 2, we can see that the complexity of these two algorithms are determined by (24) and (42) respectively. There are linear constraints, SOC constraints and Positive Semidefinite Definite(PSD) constraints in these two problems, where the linear constraint can be equivalent to one-dimension PSD constraint. According to [31,32], we know the complexity of solving an optimization problem in the worst case through the method of interior point is $\mathrm{O}(\sqrt{\theta} L \log (1 / \omega))$. Among them, $\omega$ is the precision of solutions, $\mathrm{O}(\sqrt{\theta} \log (1 / \omega))$ is the number of iteration used to find the optimal solutions, $\mathrm{O}(L)$ is the expenditure of each iteration. When an optimization problem has SOC constraints and PSD constraints, its barrier parameter is

$$
\theta=\sum_{i=1}^{m_{\text {sdp }}} k_{i, \text { sdp }}+2 m_{\text {socp }}
$$

where $m_{\text {sdp }}, k_{i, s d p}$ and $m_{\text {socp }}$ are the number of PSD constraints, dimension in $i$-th PSD constraint and SOC constraints respectively. The expenditure of each computation, i.e., $\mathrm{O}(L)$, is the order of 


$$
L=n_{o p} \sum_{i=1}^{m_{\text {sp }}} k_{i, \mathrm{sdp}}^{3}+n_{o p}^{2} \sum_{i=1}^{m_{\text {sdp }}} k_{i, \mathrm{sdp}}^{2}+n_{o p} \sum_{i=1}^{m_{\text {socp }}} k_{i, \text { socp }}^{2}+n_{o p}^{3}
$$

where $n_{\text {op }}$ is the number of optimization variables, $k_{i, \text { socp }}$ is the dimensions of $i$-th SOC constraint.The number and dimension of variables and constraints in (24), (32) are summarized in Table 3. It should be pointed that the linear constraint is seen as a one-dimension PSD constraint in this table. From $\mathrm{O}(\sqrt{\theta} L \log (1 / \omega))$, we know the complexity of two algorithms under the same precision is determined by $\theta$ and $L$. Based on (44), we obtain the fifth column in Table 3 and we can see Algorithm 2 has greater barrier parameter than Algorithm 1. Furthermore, from the second column to the fourth column and combining (44), we know $\mathrm{O}(L)$ of Algorithm1 and Algorithm 2 are mainly determined by $M^{3}$ and $M^{4}$ respectively. Therefore, Algorithm 2 has higher complexity than Algorithm 1 in general. In other words, if we can not judge multiple Eves wiretap confidential information in which way, using Algorithm 2 may guarantee the security of system more stably. But the complexity of algorithm also increases. So we should select corresponding algorithm based on the resource condition of system.

Table 3. Analysis of algorithm 1 and algorithm 2

\begin{tabular}{|c|c|c|c|c|}
\hline Algorithm & $\begin{array}{l}\text { Variable } \\
\text { (dimension,numb } \\
\text { er) }\end{array}$ & $\begin{array}{l}\text { PSD constraint } \\
\text { (dimension, } \\
\text { number) }\end{array}$ & $\begin{array}{l}\text { SOCconstraint } \\
\text { (dimension, } \\
\text { number) }\end{array}$ & $\begin{array}{l}\text { barrier parameter } \\
\qquad \theta\end{array}$ \\
\hline $\begin{array}{l}\text { Algorithm } \\
1\end{array}$ & $\begin{array}{c}\left(N_{M}, M\right) \\
\left(N_{F}, N K\right) \\
(1,6)\end{array}$ & $(1,3+N+M+N K+2 B)$ & $(1,1)$ & $N+M+N K+2 B+5$ \\
\hline $\begin{array}{l}\text { Algorithm } \\
\quad 2\end{array}$ & $\begin{array}{c}\left(N_{M}, M\right) \\
\left(N_{F}, N K\right) \\
(1,1)\end{array}$ & $\begin{array}{c}(1,2 M+N K+N+2) \\
\left(N_{M}, M\right) \\
\left(N_{F}, N K\right)\end{array}$ & 0 & $\begin{aligned} N+\left(2+N_{M}\right) M & +\left(N_{F}+1\right) N K \\
& +2\end{aligned}$ \\
\hline
\end{tabular}

\section{Simulation Results and Analysis}

In this section, we compare proposed scheme with two compared schemes respectively. In compared scheme 1, the beamforming vectors of MBS and cooperative FBSs are optimized jointly to maximize the transmission rate of $\mathrm{MU}_{1}$ while guaranteeing the QoS of other legitimate users [33]. In compared scheme 2, there is no cooperation between MBS and FBSs. In this scheme, all the FBSs are assigned to frequency resource which is orthogonal to that of MBS and only the beamforming vectors of MBS are optimized and MBS transmits random artificial noise. All the simulation results are obtained averagely through 1000 Monte Cario simulations and the channel is modeled as Rayleigh fading channel. $\varepsilon=0$ represents the perfect CSI case, while $\varepsilon>0$ represents the imperfect CSI case. Due to great differences in obtaining the optimal beamforming vectors in two eavesdroppers scenarios, the influence of same variable on secrecy performance is shown in two figures rather in the same figure, such as Fig. 2 and Fig. 5, Fig. 3 and Fig. 6. 


\subsection{Non-colluding Eves scenario}

In the simulations of this section, we assume that there are $N_{M}=8, N_{F}=4, M=3, K=2$ and $N=2$, respectively. Moreover, to simplify the analysis, we assume the uncertainty bound of all channel error in imperfect CSI case is uniform. The influence of $P_{M}$ on secrecy rate of system is shown in Fig. 2, wherein $B=3$ and $P_{F}=35 \mathrm{dBm}$. As is shown in Fig. 2, under the same condition, the secrecy performance in the proposed scheme is always superior to that of two compared schemes with the increase of $P_{M}$. It is because, compared scheme 1 ignores the coupling of improving the transmission rate and the leakage of confidential information, which also leads to more information leakage for Eves. In compared scheme 2, random artificial noise jams Eves and $\mathrm{MU}_{1}$ in the same extent and it does not introduce the downlink signal of cooperative FBSs to deteriorate the received performance of Eves more severely. Different from two compared schemes, the proposed scheme considers the coupling of improving the transmission rate and the leakage of confidential information. So the transmission rate of $\mathrm{MU}_{1}$ is improved and Eves suffers from more severe interference through optimizing the beamforming vectors of MBS and cooperative FBSs in proposed scheme. In fact, the interference from the downlink signal of other legitimate users in proposed scheme can be seen as "natural noise". Moreover, from Fig. 2, we can see that proposed scheme always has better secrecy performance than two compared schemes under the same channel error, and the channel error has smaller influence on proposed scheme, which shows the proposed scheme has better robustness.

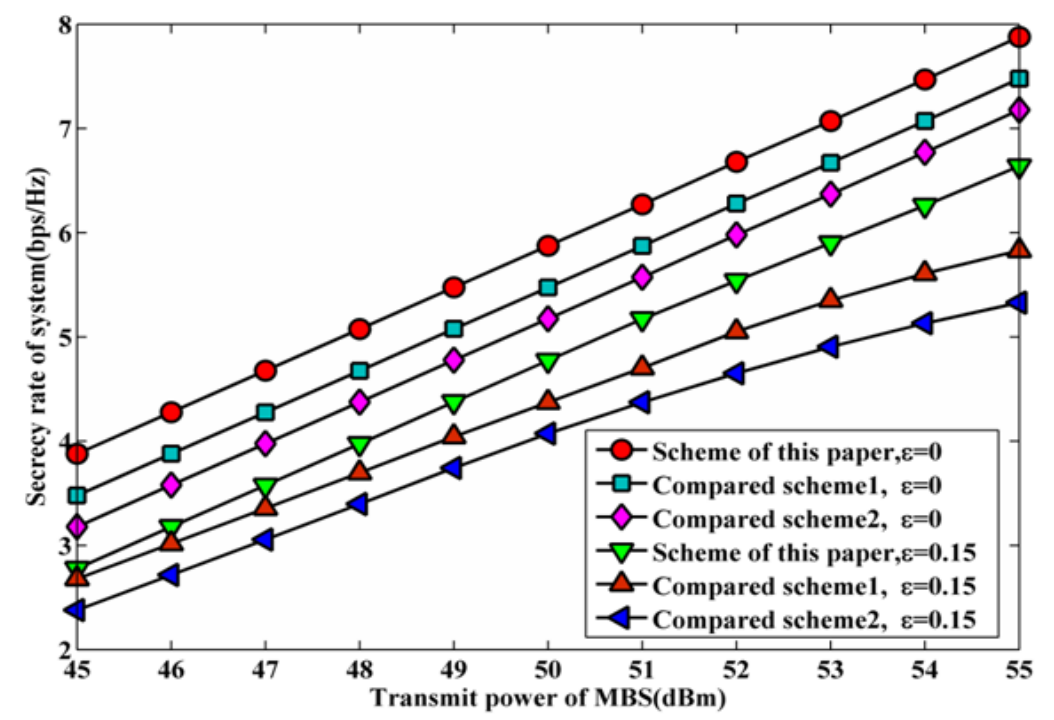

Fig. 2. Influence of $P_{M}$ on secrecy rate of system in non-colluding Eves scenario

The influence of on $P_{F}$ secrecy rate of system is shown in Fig. 3, wherein $B=3$ and $P_{M}=40 \mathrm{dBm}$. In compared scheme 2, there is no cooperative FBSs, so Fig. 3 only shows the comparison between proposed scheme and compared scheme 1. As is shown in Fig. 3, the secrecy rate of system rises with the increase of $P_{M}$ in proposed scheme and is always greater than that of compared scheme 1. It is because that the interference signal of cooperative FBSs can jam Eves more severely through jointly optimizing the beamforming vectors and smaller 
distance between cooperative FBSs and Eves. Similarly, from Fig. 3, we can see that the same channel error has smaller influence on proposed scheme. Therefore, when $P_{M}$ is fixed, Eves will received more interference through increasing the $P_{F}$ or $N$.

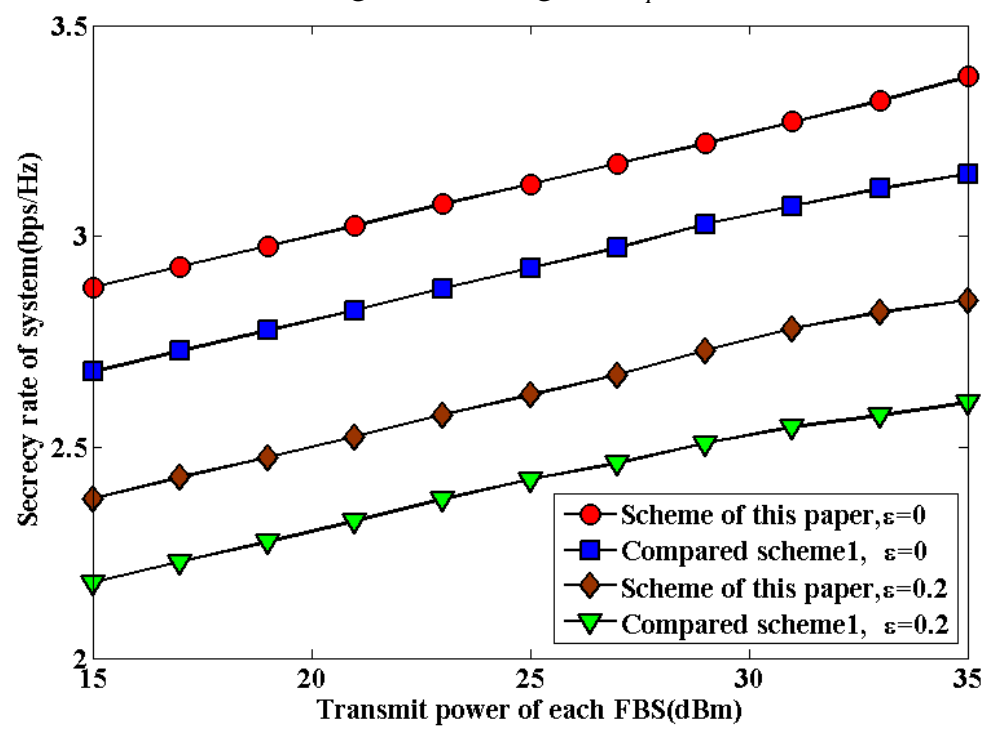

Fig. 3. Influence of $P_{F}$ on secrecy rate of system in non-colluding Eves scenario

The convergence of algorithm 1 is shown in Fig. 4, wherein $P_{M}=45 \mathrm{dBm}$ and $P_{F}=35$ $\mathrm{dBm}$. As is shown in Fig. 4, the secrecy rate of system increases monotonously and is capable of reaching a stable value within 4 iterations, which proves the correctness of analysis in 3.1.2. It is also observed that the secrecy rate of system decreases as the number of Eves increases, which means the increase of the number of Eves will pose greater danger for system security. Additionally, both the number of Eves and the channel error have no influence on the convergence speed of algorithm 1 .

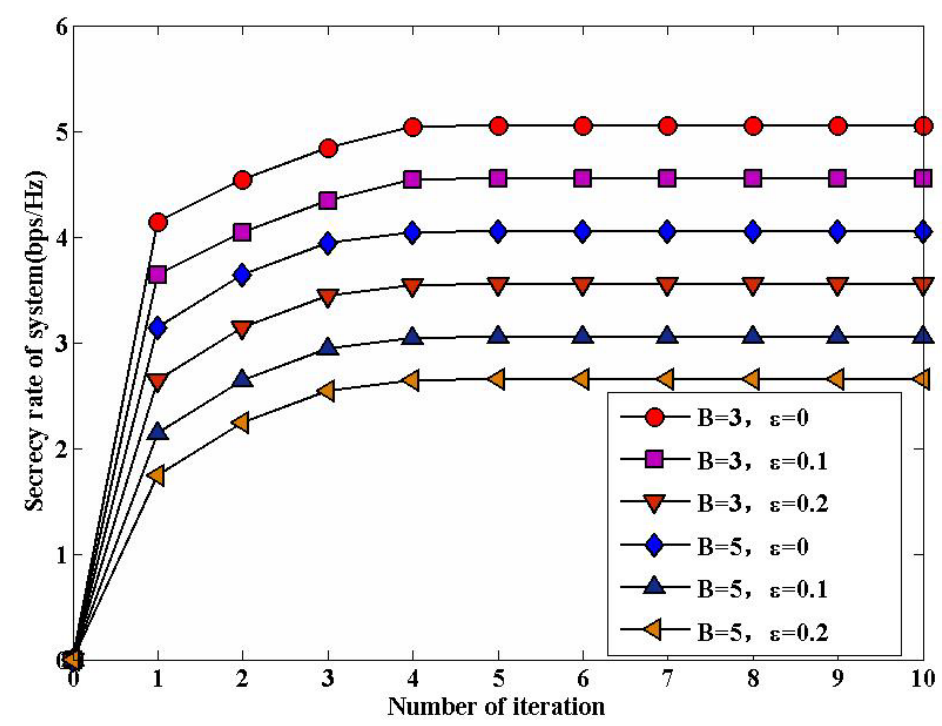

Fig. 4. Convergence of algorithm 1 


\subsection{Colluding Eves scenario}

In the simulations of this section, we assume that there are $N_{M}=10, N_{F}=4, M=3, K=2$ and $N=4$ respectively. Similarly, to simplify the analysis, we assume the uncertainty bound of channel error in imperfect CSI scenario is uniform and $\Gamma^{\prime}=0.5$. Fig. 5 shows the comparison between proposed scheme and two compared schemes, wherein $P_{F}=35 \mathrm{dBm}$ and $\varepsilon=0$. As is shown in Fig. 5, the secrecy rate of system decreases when the number of Eves increases. Because they will acquire more confidential information. With the increase of $P_{M}$, the secrecy performance of proposed scheme is always superior to that of two compared schemes for the same condition. The reason for this phenomenon is similar to that in above section. So we will not repeat it in this section for concision.

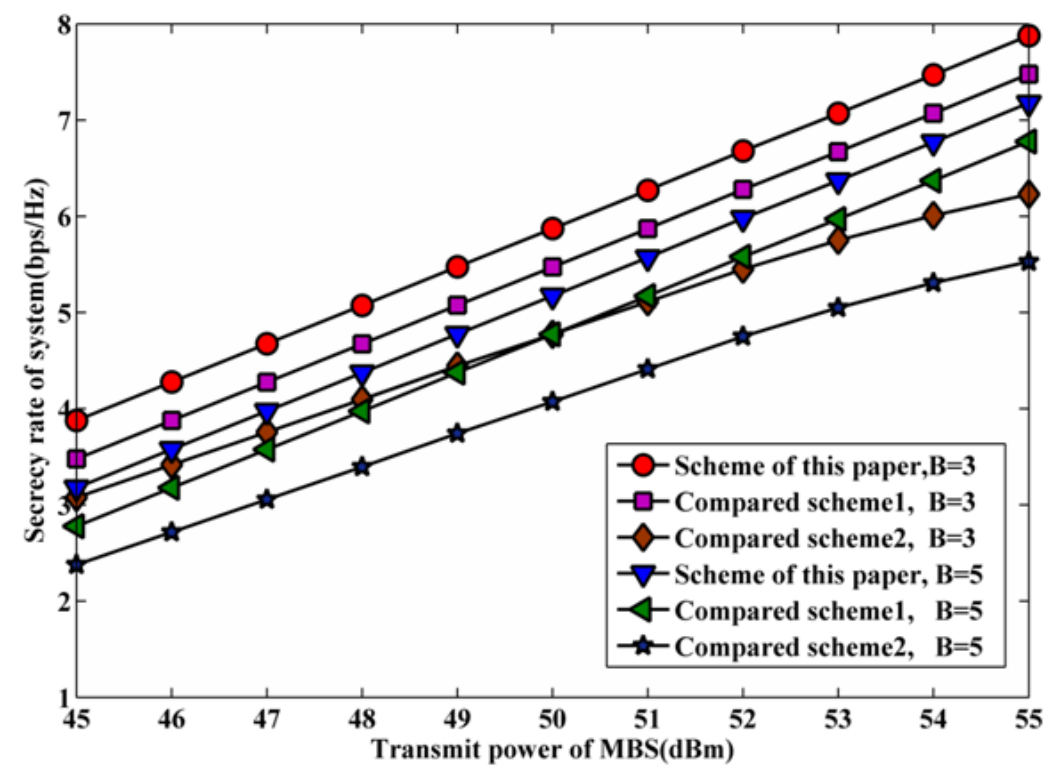

Fig. 5. Influence of $P_{M}$ on the secrecy rate of system in colluding Eves scenario

Fig. 6 shows the comparison between proposed scheme and compared scheme 1, wherein $P_{M}=40 \mathrm{dBm}$ and $\varepsilon=0$. As is shown in Fig. 6, as the transmitted power of each cooperative FBS increases, the secrecy rate of proposed scheme is always superior to compared scheme 1. Similarly, the reason for this phenomenon is similar to that in above section. So we will not repeat it. 


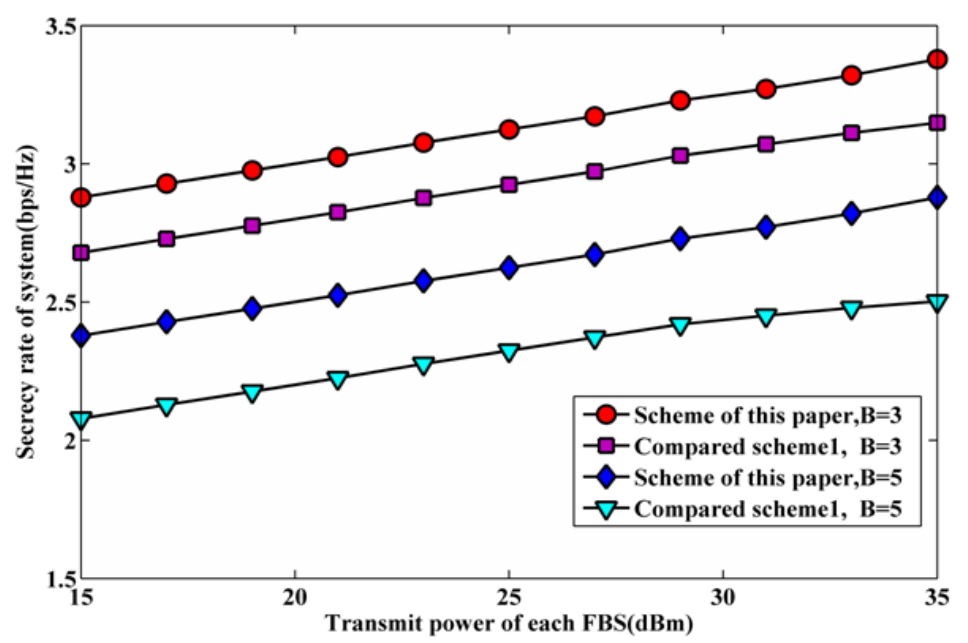

Fig. 6. Influence of $P_{F}$ on the secrecy rate of system in colluding Eves scenario

Fig. 7 shows the influence of $B$ on the secrecy rate of system, wherein $P_{M}=55 \mathrm{dBm}$, $P_{F}=35 \mathrm{dBm}$ respectively. As is shown in Fig. 7, the secrecy rate of system decreases when the number of Evesincreases. It is because with the rise of the number of Eves, they will have more antennas and its wiretapped performance will also improve, which proves the correctness of analysis in section 2. It is also observed that the secrecy rate of proposed scheme is always superior to that of two compared schemes in the same channel error condition, which shows the security and robustness of proposed scheme.

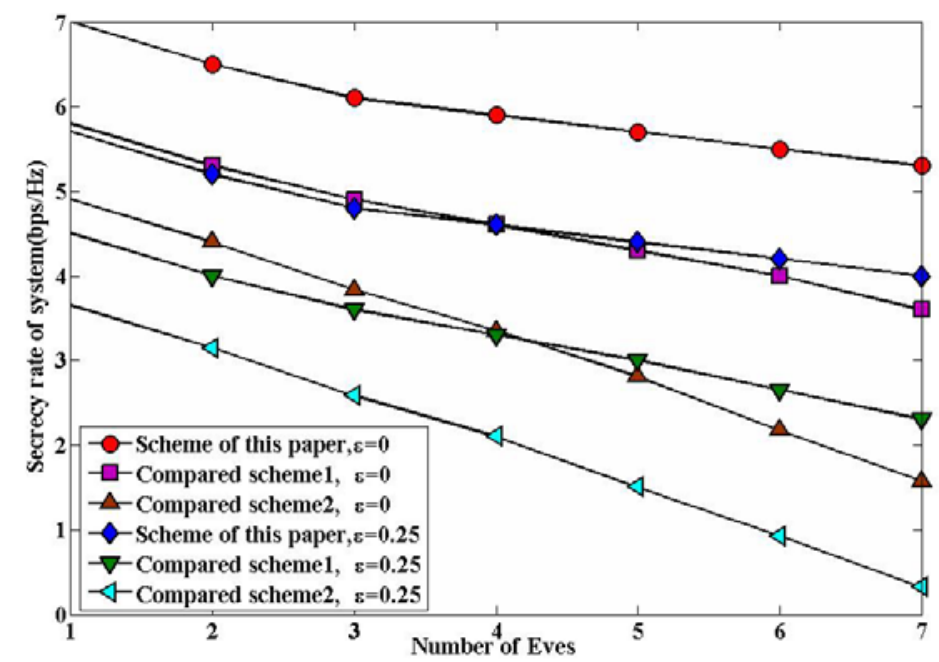

Fig. 7. Influence of $B$ on the secrecy rate of system in colluding Eves scenario

\section{Conclusion}

In this paper, we have explored a physical layer security scheme based on cross-layer cooperation in a two-tier downlink dense heterogeneous network. Through introducing the 
downlink signals of other legitimate users as the interference for Eves, and further jointly optimizing the beamforming vectors of MBS and cooperative FBSs, we can maximize the secrecy rate of system. Based on the relationship between multiple Eves, we investigate the problem of secrecy rate maximization in two scenarios respectively. In non-colluding Eves scenario, we solve the originally non-convex optimization problem through SDR technique and SCA algorithm to obtain the optimal bemaforming vectors in perfect CSI case. And we extend the frame to imperfect CSI case. Then in colluding Eves scenario, we transform the original optimization problem into a two-tier optimization problem through equivalent transformation. Finally, we analyze and compare the complexity of proposed algorithms to obtain optimal solutions in two scenarios. Simulation results validate the effectiveness and robustness of proposed scheme in this paper.

\section{References}

[1] Zhang H, Liu H, and Cheng J, "Downlink Energy Efficiency of Power Allocation and Wireless Backhaul Bandwidth Allocation in Heterogeneous Small Cell Networks," IEEE Transactions on Communications, to be published. Article (CrossRef Link).

[2] Li B, Fei Z, Chu Z, "Secure Transmission for Heterogeneous Cellular Networks with Wireless Information and Power Transfer," IEEE Systems Journal, to be published.

Article (CrossRef Link).

[3] H. Zhang, S. Huang, and C. Jiang, "Energy Efficient User Association and Power Allocation in Millimeter Wave Based Ultra Dense Networks with Energy Harvesting Base Stations," IEEE Journal on Selected Areas in Communications, vol. 35, no. 9, pp. 1936-1947, September, 2017. Article (CrossRef Link).

[4] Li B, Fei Z and Chu Z, "Optimal Transmit Beamforming for Secure SWIPT in a Two-Tier HetNet,” IEEE Communications Letters, vol. 21, no. 11, pp. 2476-2479, November, 2017. Article (CrossRef Link).

[5] Zhang X N, Tan Z H and Xu S Y, "Utility maximization based on cross-layer design for multi-service in macro-femto heterogeneous networks," IEEE Transactions on Wireless Communication, vol. 12, pp. 5607-5620, November, 2013. Article (CrossRef Link).

[6] Li N, Tao X and Wu H, "Large-System Analysis of Artificial-Noise-Assisted Communication in the Multiuser Downlink: Ergodic Secrecy Sum Rate and Optimal Power Allocation,” IEEE Transactions on Vehicular Technology, vol. 65, no. 9, pp. 7036-7050, September, 2016. Article (CrossRef Link).

[7] Li N, Tao X and Xu J, "Ergodic Secrecy Sum-Rate for Downlink Multiuser MIMO Systems With Limited CSI Feedback,” IEEE Communications Letters, vol. 18, no. 6, pp.969-972, June, 2014. Article (CrossRef Link).

[8] Zhang H, Xing H, and Cheng J, "Secure Resource Allocation for OFDMA Two-Way Relay Wireless Sensor Networks Without and With Cooperative Jamming," IEEE Transactions on Industrial Informatics, vol. 12, no. 5, pp. 1714-1725, Octember, 2017. Article (CrossRef Link).

[9] Zheng C, Zhu Z and Hussein J, "Robust Optimization for AN-Aided Transmission and Power Splitting for Secure MISO SWIPT System,” IEEE Communications Letters, vol. 20, no. 8, pp. 1571-1574, August, 2016. Article (CrossRef Link).

[10] Zhong Zhi-hao, Peng Jian-hua and Luo Wen-yang, "A Tractable Approach to Analyzing the Physical-Layer Security in K-Tier Heterogeneous Cellular Networks," China Communications, vol. 12, no. 1, pp. 166-173, December, 2015. Article (CrossRef Link).

[11] Wang H M, Zheng T X and Yuan J, "Physical layer security in heterogeneous cellular networks," IEEE Transactions on Communications, vol. 64, no. 3, pp. 1204-1219, March, 2016. Article (CrossRef Link).

[12] Wu H, Tao X and Li N, "Secrecy Outage Probability in Multi-RAT Heterogeneous Networks," IEEE Communications Letters, vol. 20, no. 1, pp. 53-56, January, 2016. Article (CrossRef Link). 
[13] Zhong Zhi-hao, Luo Wen-yu, and Peng Jian-hua, "Secrecy Performasnce Analysis of Cooperative Transmisson and Cooperative jamming in K-tier Heterogeneous Cellular Networks,” Science China: Information Science, vol. 1, pp. 004-011, January, 2016. Article (CrossRef Link).

[14] Xu M, Tao X and Yang F, "Enhancing Secured Coverage with CoMP Transmission in Heterogeneous Cellular Networks,” IEEE Communications Letters, vol. 20, no. 11, pp. 2272-2275, November, 2016. Article (CrossRef Link).

[15] Liu Y, Chen H H and Wang L, "Physical Layer Security for Next Generation Wireless Networks: Theories, Technologies, and Challenges," IEEE Transactions on Information Forensics and Security, vol. 19, no. 1, pp. 347-376, August, 2017. Article (CrossRef Link).

[16] Y Wang, Z Miao and L Jiao, "Safeguarding the Ultra-dense Networks with the aid of Physical Layer Security: A review and a case study,” IEEE Access, vol. 4, no. 99, pp. 9082-9092, December, 2017. Article (CrossRef Link).

[17] LUO Z Q, MA W K and SO A C, "Semidefinite relaxation of quadratic optimization problems," IEEE Signal Processing Magazine, vol. 27, no. 3, pp. 20-34, April, 2010. Article (CrossRef Link).

[18] S. Yang, T. Lv, and L. Hanzo, "Semidefinite programming relaxation based virtually antipodal detection for MIMO systems using Gray-coded high-order QAM," IEEE Transactions on Vehicular Technology, vol. 62, no. 4, pp. 1667-1677, December, 2013. Article (CrossRef Link).

[19] BECK A, BEN-TAL A and TETRUASHVILI L, "A sequential parametric convex approximation method with applications to nonconvex truss topology design problems," Journal of Global Optimization, vol. 47, no. 1, pp. 29-51, May, 2010. Article (CrossRef Link).

[20] CHARNES A, COOPER W, "Programming with linear fractional functionals," Naval Research Logistics Quarterly, vol. 9, no. 3, pp. 181-186, March, 2010. Article (CrossRef Link).

[21] Y Ren, T Lv and H Gao, "Secure Wireless Information and Power Transfer in Heterogeneous Networks,” IEEE Access, vol. 5, no. 99, pp. 4967-4979, June, 2017. Article (CrossRef Link).

[22] Lv T, Gao H and Cao R, "Coordinated Secure Beamforming in K-User Interference Channel with Multiple Eavesdroppers,” IEEE Wireless Communication Letters, vol. 5, no. 2, pp. 212-215, January, 2016. Article (CrossRef Link).

[23] Y.-S. Shiu, S.-Y and Chang.-H, "Physical layer security in wireless networks: A tutorial," IEEE Wireless Communication, vol. 18, no. 2, pp. 66-74, April, 2011. Article (CrossRef Link).

[24] Zhang Li-jian, Jin Liang and Luo Wen-yu, "Secure coordinated beamforming for multiuser MISO interference channels,” Journal on Communications, vol. 36, no. 11, pp. 41-51, November, 2015. Article (CrossRef Link).

[25] D. Lopez-Perez, "Enhanced intercell interference coordination challenges in heterogeneous networks,” IEEE Wireless Communication, vol. 18, no. 3, pp. 22-30, June, 2011. Article (CrossRef Link).

[26] WAJID I, PESAVENTO M and ELDAR Y C, "Robust downlink beamforming with partial channel state information for conventional and cognitive radio networks," IEEE Transactions on Signal Processing, vol. 61, no. 14, pp. 3656-3670, May, 2013. Article (CrossRef Link).

[27] Ponukumati D, Gao F and Bode M, "Robust coordinated downlink beamforming for multicell-cognitive radio networks,” in Proc. of IEEE 24th Annual International Symposium on Personal, Indoor, and Mobile Radio Communications (PIMRC), pp. 719-723, September 8-11, 2013. Article (CrossRef Link).

[28] Nasseri S, Le T A and Nakhai M R, "Robust and power efficient interference management in downlink multi-cell networks," in Proc. of IEEE 24th Annual International Symposium on Personal, Indoor, and Mobile Radio Communications (PIMRC), pp. 1097-1101, September 8-11, 2013. Article (CrossRef Link).

[29] WAJID I, ELDAR Y C and GERSHMAN A B, "Robust downlink beamforming for cognitive radio networks,” in Proc. Of GLOBECOM'10, Florida, USA, pp. 1254-1258, December 6-10, 2010. Article (CrossRef Link).

[30] Z.-Q. Luo, W.-K. Ma and A. M.-C, "Semidefinite relaxation of quadratic optimization problems,’’ IEEE Signal Processing Magzine, vol. 27, no. 3, pp. 20-34, April, 2010. Article (CrossRef Link). 
[31] Zhang L, Jin L, Luo W, "Robust secure transmission for multiuser MISO systems with probabilistic QoS constraints,” Science China Information Sciences, vol. 59, no. 2, pp.1-13, January, 2016. Article (CrossRef Link).

[32] Li B, Fei Z, "Probabilistic-constrained robust secure transmission for energy harvesting over MISO channels,” Science China Information Sciences, vol. 61, no. 2, pp. 1-12, February, 2018. Article (CrossRef Link).

[33] S. A. A. Fakoorian and A. L. Swindlehurst, "MIMO interference channel with confidential messages: Achievable secrecy rates and precoder design,” IEEE Transactions on Information Forensics and Security, vol. 6, no. 3, pp. 640-649, May, 2011. Article (CrossRef Link).

[34] Zhu F, Yao M, "Improving Physical Layer Security for CRNs Using SINR-Based Cooperative Beamforming,” IEEE Transactions on Vehicular Technology, vol. 1, no. 1, pp. 1-7, March, 2016. Article (CrossRef Link).

\section{Appendix}

\section{Proof of proposition 1}

The problem (14) is equivalent to the following problem

$$
\begin{gathered}
\min \left\{\operatorname{Tr}\left(\sum_{m=1}^{M} \boldsymbol{W}_{m}+\sum_{n=1}^{N} \sum_{k=1}^{K} \boldsymbol{W}_{n k}\right)\right\} \\
\text { s.t. constraint }(15 \mathrm{~b}) \sim(15 \mathrm{e}) \\
\operatorname{Tr}\left(\boldsymbol{H}_{1} \boldsymbol{W}_{1}\right) \geq\left(t_{1}-1\right)\left(\sum_{m=2}^{M} \operatorname{Tr}\left(\boldsymbol{H}_{1} \boldsymbol{W}_{m}\right)+\sum_{n=1}^{N} \sum_{k=1}^{K} \operatorname{Tr}\left(\boldsymbol{H}_{n, 1} \boldsymbol{W}_{n k}\right)+1\right) \\
\operatorname{Tr}\left(\boldsymbol{H}_{E b} \boldsymbol{W}_{1}\right) \leq\left(\frac{1}{t_{2}}-1\right)\left(\sum_{m=2}^{M} \operatorname{Tr}\left(\boldsymbol{H}_{E b} \boldsymbol{W}_{m}\right)+\sum_{n=1}^{N} \sum_{k=1}^{K} \operatorname{Tr}\left(\boldsymbol{H}_{n, E b} \boldsymbol{W}_{n k}\right)+1\right)
\end{gathered}
$$

Utilizing the Lagrange Multiplier [34], we can transform the above problem as

$$
L\left(\alpha^{\wedge}, \alpha_{n}^{\wedge}, \beta_{m}^{\wedge}, \lambda_{n k}, u^{\wedge}, \varphi^{\wedge}\right)=\operatorname{Tr}\left(\boldsymbol{D}_{1}^{\wedge} \boldsymbol{W}_{1}\right)+\operatorname{Tr}\left(\boldsymbol{D}_{m}{ }^{\wedge} \boldsymbol{W}_{m}\right)+\operatorname{Tr}\left(\boldsymbol{D}_{n k}{ }^{\wedge} \boldsymbol{W}_{n k}\right)+C^{\wedge}
$$

where $\alpha^{\wedge} \geq 0,\left\{\alpha_{n} \geq 0\right\},\left\{\beta_{m}^{\wedge} \geq 0\right\},\left\{\lambda_{n k} \wedge 0\right\}, u^{\wedge} \geq 0, \varphi^{\wedge} \geq 0$ denote the dual variables of problem A associated with the constraints in (A1) (A7), respectively, and

$$
\begin{aligned}
\boldsymbol{D}_{1}^{\wedge} & =\left(\alpha^{\wedge}+1\right) \boldsymbol{I}+\varphi^{\wedge} \sum_{b=1}^{B} \boldsymbol{H}_{E b}+\sum_{m=2}^{M} \beta_{m}^{\wedge} \gamma_{m} \boldsymbol{H}_{m}+\sum_{n=1}^{N} \sum_{k=1}^{K} \lambda_{n k} \gamma_{n k} \boldsymbol{H}_{n k}-\mu^{\wedge} \boldsymbol{H}_{1} \\
\boldsymbol{D}_{m}{ }^{\wedge}=\left(\alpha^{\wedge}+1\right) \boldsymbol{I} & +\sum_{p \neq 1, m}^{M} \beta_{p}^{\wedge} \gamma_{p} \boldsymbol{H}_{p}+\sum_{n=1}^{N} \sum_{k=1}^{K} \lambda_{n k}{ }^{\wedge} \gamma_{n k} \boldsymbol{H}_{n k}+\mu^{\wedge}\left(t_{1}-1\right) \boldsymbol{H}_{m}+\varphi^{\wedge}\left(\frac{1}{t_{2}}-1\right) \sum_{b=1}^{B} \boldsymbol{H}_{E b}-\beta_{m}{ }^{*} \boldsymbol{H}_{m}, m \neq 1 \\
\boldsymbol{D}_{n k} \wedge & \left(1+\sum_{n=1}^{N} \alpha_{n}{ }^{\wedge}\right) \boldsymbol{I}+\sum_{m=2}^{M} \beta_{m}^{\wedge} \gamma_{m} \boldsymbol{H}_{n, m}+\sum_{t \neq k}^{K} \lambda_{n t} \wedge \gamma_{n t} \boldsymbol{H}_{n, n t} \\
& +\sum_{a \neq n}^{N} \sum_{k=1}^{K} \lambda_{a k} \gamma_{a k} \boldsymbol{H}_{n, a k}+\mu^{\wedge}\left(t_{1}-1\right) \boldsymbol{H}_{n, 1}+\varphi\left(\frac{1}{t_{2}}-1\right) \sum_{b=1}^{B} \boldsymbol{H}_{n, E b}-\lambda_{n k}{ }^{*} \boldsymbol{H}_{n, n k}
\end{aligned}
$$

According to analysis, there are $t_{1}-1>0$ and $1 / t_{2}>0$. Since $\operatorname{Rank}\left(\boldsymbol{H}_{1}\right) \leq 1$ and $\operatorname{Rank}\left(\left(\alpha^{\wedge}+1\right) \boldsymbol{I}+\varphi^{\wedge} \sum_{b=1}^{B} \boldsymbol{H}_{E b}+\sum_{m=2}^{M} \beta_{m}^{\wedge} \gamma_{m} \boldsymbol{H}_{m}+\sum_{n=1}^{N} \sum_{k=1}^{K} \lambda_{n k} \wedge \gamma_{n k} \boldsymbol{H}_{n k}\right)=N_{M}$, the following solution can be derived

$$
\operatorname{Rank}\left(\boldsymbol{D}_{1}^{\wedge}\right) \geq N_{M}-1
$$


Similar to $\boldsymbol{D}_{1}^{\wedge}$, we can obtain two solutions, i.e., $\operatorname{Rank}\left(\boldsymbol{D}_{m}{ }^{\wedge}\right) \geq N_{M}-1$, $\operatorname{Rank}\left(\boldsymbol{D}_{n k}{ }^{\wedge}\right) \geq N_{F}-1$. Then, according to the Karush-Kuhn-Tucker(KKT) [34] conditions, there are following derivations

$$
\boldsymbol{D}_{1}^{\wedge} \boldsymbol{W}_{1}^{\wedge}=0, \quad \boldsymbol{D}_{m}^{\wedge} \boldsymbol{W}_{m}^{\wedge}=0, \quad \boldsymbol{D}_{n k}{ }^{\wedge} \boldsymbol{W}_{n k}{ }^{\wedge}=0
$$

Combining (A12) and (A13), we get these derivations, i.e., $\operatorname{Rank}\left(\boldsymbol{W}_{1}^{\wedge}\right) \leq 1, \operatorname{Rank}\left(\boldsymbol{W}_{m}^{\wedge}\right) \leq 1$, $\operatorname{Rank}\left(\boldsymbol{W}_{n k}{ }^{\wedge}\right) \leq 1$. When $\operatorname{Rank}\left(\boldsymbol{W}_{1}^{\wedge}\right)=0$, the transmission rate of eavesdropped MU is zero. We can not get the optimal solutions in this case. When $\operatorname{Rank}\left(\boldsymbol{W}_{m}{ }^{\wedge}\right)=0$, the QoS requirements of other MUs can not be satisfied, which go against with the constraint (23d). Similarly, $\operatorname{Rank}\left(W_{n k}{ }^{\wedge}\right)=1$ is not correct.Therefore, the optimal beamforming matrixs must be rank of one.

\section{Proof of proposition 2}

The problem (43) is equivalent to the following problem

$$
\begin{aligned}
& \min _{\substack{\left\{w_{m}\right\}^{M} \\
\left\{\left\{=1 \\
\left\{w_{n k}\right\}_{k=1}^{N}\right\}_{n=1}^{N}\right.}} \Gamma\left(\sum_{b=1}^{B} \operatorname{Tr}\left(\boldsymbol{H}_{E b} \boldsymbol{X}_{1}\right)\right) \\
& \text { s.t constraint }(43 \mathrm{~b}) \sim(43 \mathrm{~h})
\end{aligned}
$$

Similar to the proof of proposition 1 , we can prove the optimal beamforming matrixs must be rank of one, i.e., $\operatorname{Rank}\left(\boldsymbol{X}_{1}^{*}\right)=\operatorname{Rank}\left(\boldsymbol{X}_{m}{ }^{*}\right)=\operatorname{Rank}\left(\boldsymbol{X}_{n k}{ }^{*}\right)=1$. 


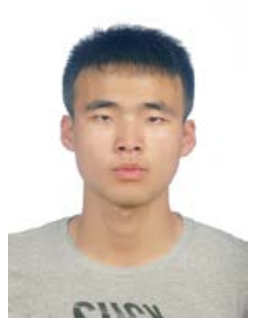

Zhang Bo received the B.E. and M.S. degrees in University of Harbin Institute of Technology and National Digital Switching System Engineering \& Technological R\&D Center (NDSC) in China, respectively. He is currently a Ph.D. candidate at NDSC, Zhengzhou, China. His research interests include physical layer security in wireless networks and cooperative wireless networks.

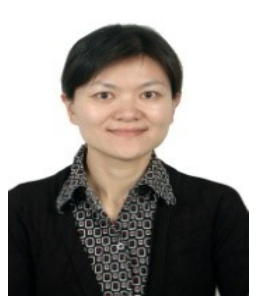

Huang Kai-zhi received her B.E. degree in digital communication and M.S. degree in communication and information system from PLA College of Information Engineering, Zhengzhou, China, and Ph.D. degrees in communication and information system from Tsinghua University, Beijing, China, in1995, 1998 and 2003 respectively. She has been a faculty member of NDSC since 1998, where she is currently a professor and director of Laboratory of Mobile Communication Networks. Her research interests include wireless network security and signal processing.

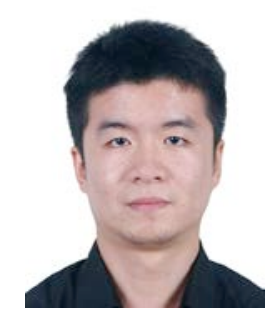

Chen Ya-jun received the B.E. and M.S. degrees in University of Electronic Science and Technology of China and NDSC, respectively. He is currently a Ph.D. candidate at NDSC, Zhengzhou, China. His research interests include physical layer security, wireless location and resource management in $5 \mathrm{G}$ networks. 